\title{
Human hepatic organoids for the analysis of human genetic diseases
}

\author{
Yuan Guan, ${ }^{1}$ Dan Xu, ${ }^{1}$ Phillip M. Garfin,, ${ }^{2,3}$ Ursula Ehmer, ${ }^{2,3}$ Melissa Hurwitz, ${ }^{2}$ Greg Enns, ${ }^{2}$ \\ Sara Michie, ${ }^{4}$ Manhong Wu, ${ }^{1}$ Ming Zheng, ${ }^{1}$ Toshihiko Nishimura, ${ }^{1,5,6}$ Julien Sage, ${ }^{2,3}$ and Gary Peltz ${ }^{1}$ \\ 'Department of Anesthesia, ${ }^{2}$ Department of Pediatrics, ${ }^{3}$ Department of Genetics, and ${ }^{4}$ Department of Pathology, \\ Stanford University School of Medicine, Stanford, California, USA. ${ }^{5}$ Center for the Advancement of Health and Bioscience, \\ Sunnyvale, California, USA. ${ }^{6}$ Central Institute for Experimental Animals, Tokyo, Japan.
}

We developed an in vitro model system where induced pluripotent stem cells (iPSCs) differentiate into 3-dimensional human hepatic organoids (HOs) through stages that resemble human liver during its embryonic development. The HOs consist of hepatocytes, and cholangiocytes, which are organized into epithelia that surround the lumina of bile duct-like structures. The organoids provide a potentially new model for liver regenerative processes, and were used to characterize the effect of different JAC1 mutations that cause: (a) Alagille syndrome (ALCS), a genetic disorder where NOTCH signaling pathway mutations impair bile duct formation, which has substantial variability in its associated clinical features; and (b) Tetralogy of Fallot (TOF), which is the most common form of a complex congenital heart disease, and is associated with several different heritable disorders. Our results demonstrate how an iPSC-based organoid system can be used with genome editing technologies to characterize the pathogenetic effect of human genetic disease-causing mutations.

Conflict of interest: The authors have declared that no conflict of interest exists.

Submitted: May 5, 2017

Accepted: July 25, 2017

Published: September 7, 2017

Reference information: JCI Insight. 2017;2(17): e94954. https://doi.org/10.1172/jci. insight.94954.

\section{Introduction}

We have obtained much information about the signaling pathways affecting the development of solid organs, such as the liver (1), from analysis of model organisms. However, a major unsolved problem is defining the role of these pathways in solid organ development in humans. The abnormalities caused by certain genetic diseases provide information about the key signaling pathways involved in human organ development. For example, Alagille syndrome (ALGS) is an autosomal dominant genetic disorder (with incomplete penetrance) that is predominantly (94\%) caused by mutations in the gene (JAG1) that codes for the NOTCH ligand JAG1 $(2,3)$. The clinical course is dominated by severe liver abnormalities, and its predominant pathologic feature is a paucity of bile ducts, which results from abnormal development of the biliary tree. A wide range of $J A G 1$ mutations, which are spread across the entire protein, have been found: approximately $80 \%$ produce protein truncations, $8 \%$ are whole-gene deletions, and $12 \%$ are missense mutations (4). There is substantial heterogeneity in the clinical features observed in subjects with $J A G 1$ mutations. For example, only 11 of 53 JAG1 mutation-positive relatives of ALGS subjects had clinical features that would lead to a diagnosis of ALGS, and 9 of the relatives had cardiac abnormalities without liver disease (5). Various cardiovascular abnormalities are also associated with ALGS. Of these, Tetralogy of Fallot (TOF) (6), which develops in 7\%-13\% of ALGS subjects (7), is of particular interest. TOF is the most common form of complex congenital heart disease; it is associated with several different heritable disorders and has the following features: ventral septal defect, overriding aorta, pulmonic stenosis, and right ventricular hypertrophy (8). JAG1 mutations have been identified in subjects with classic features of TOF who do not have liver disease (9). Although many $J A G 1$ mutations have been identified, a fundamental question about the heterogeneity of the clinical features caused by $J A G 1$ mutations remains unanswered: Is it caused by the effect of different JAG1 mutations or is it determined by other elements in the genetic background?

JAG1 is 1 of 5 Notch signaling ligands (4). NOTCH proteins are a highly conserved family of transmembrane receptors $(10)$ that play a major role in cell fate determination $(11,12)$, biliary tract development $(13,14)$, and in liver cancer $(15,16)$. Although much information has been obtained about the NOTCH signaling pathway, we do not fully understand the pathobiology of human ALGS liver disease. How does a reduction in Notch signaling hinder bile duct formation? Based on the general mechanisms of action of the Notch pathway, it is thought that decreased JAG1 expression in nonepithelial cells, which interact with bile duct cells during the for- 
mation of bile ducts, causes ALGS pathology. An intricate network of different cell types (hepatoblasts, vascular endothelial cells, portal mesenchymal cells) drives intrahepatic bile duct development in the liver (1). Hence, the identity of the JAG1-expressing cells that determine human bile duct specification remains unclear. The relative importance of JAG1 in epithelial (versus nonepithelial) cells is unknown, and a mesenchymal-epithelial cell interaction is required for biliary tract formation during embryonic development $(17,18)$. A specific deletion of the mouse $J A G 1$ gene in liver mesenchymal structures (but not in endothelium) of mouse embryos recapitulates in part the biliary defects observed in patients (19), but the animal models used to study ALGS only recapitulate certain aspects of the syndrome $(19,20)$. As examples, deletion of the entire Jag1 gene in mutant mice does not model the point mutations found in patients, and $\operatorname{Jag} 1^{+/-}$mice $(21,22)$ do not develop the liver features of ALGS, while heterozygous humans do. Mutant mice display very homogeneous phenotypes, while there is substantial variability in ALGS patients. Recently, a $\mathrm{Jag} 1^{+/-}$mouse generated on a particular genetic background was found to develop bile duct paucity (23). However, their livers had extensive focal inflammatory lesions and ductular reactions, which are not present in human ALGS liver in subjects of the same age, and these could be more characteristic of another biliary tract disease (biliary atresia) (7).

We do not completely understand whether JAG1 mutations cause ALGS liver pathology by a dominant-negative or a haploinsufficiency mechanism. Because chromosomal deletions at 20p12 were present in some ALGS subjects (24-26), it was initially proposed that ALGS liver disease was caused by a gene deletion (haploinsufficiency). When the causative $J A G 1$ mutations were initially characterized $(2,3)$, the investigators definitively "conclude that ALGS is caused by haploinsufficiency of JAG1" (3). In more recent analyses of a much larger number of ALGS subjects $(27,28)$, the appearance of a cholangiopathy in $J A G 1$ heterozygous-knockout mice (23) and the characterization of the properties of an expressed mutated JAG1 protein were also all interpreted as indicating that haploinsufficiency was the causative mechanism (29). However, many ALGS subjects have point mutations (instead of a gene deletion), so it was also possible that some mutations could act via a dominant-negative effect $(27,29)$. To more fully understand the pathogenesis of this genetic disease, we must have a system that more closely accounts for the genetic impact that ALGS mutations have on human liver development. Here, we developed a method for producing hepatic organoids from human induced pluripotent stem cells (iPSCs). We then analyzed these organoids to answer fundamental questions about how genetic mutations can cause ALGS liver disease and about the heterogeneity in the clinical features associated with $J A G 1$ mutations.

\section{Results}

Generation of human hepatic organoids from iPSCs. To characterize the mechanisms affecting liver and bile duct development, a culture system was developed to differentiate iPSCs into hepatic organoids (HOs) (Figure 1A). Detailed information about the development of this protocol is provided in a supplemental note. In brief, iPSCs were first dissociated into single cells and induced to form endoderm spheres in response to growth factors and chemicals added to the culture according to a modified protocol (30). The endodermal spheres then formed posterior foregut-like structures when cultured in the presence of a low concentration $(1 \%-2 \%)$ of a Matrigel scaffold that supports the formation of $3 \mathrm{D}$ structures from stem cells $(31,32)$, and by addition of various concentrations of FGF10, which is known to promote the differentiation of foregut endoderm into hepatic and gallbladder cells during organogenesis (33). The developing foregut structures were transferred on day 9 into a medium with other growth factors to promote their maturation into HOs. By day 20, the organoids developed a more complex structure that we refer to as primary HOs (HO1s), which could be seen in stereomicroscopic images (Figure 1B). The proliferative and regenerative potential of HO1s may be limited when they reach a 3-mm diameter, because it is difficult for them to obtain sufficient nutrients and oxygen. To overcome this limitation, the HO1 cells were dissociated into single cells, embedded in Matrigel, and then cultured for 6 days in a growth medium that promoted their proliferation. The cells were then cultured for another 6 days in a medium to promote their differentiation into HOs. Remarkably, the dissociated cells could reform organoids, which we refer to as secondary HOs (HO2s) (Figure 1B), and these are fully characterized in the liver regeneration section below.

We examined the pattern of protein and mRNA expression in differentiating $\mathrm{HO}$ cultures. The endodermal spheres were shown by immunostaining (SOX17 and CXCR4) to express endodermal lineage markers on day 3 (Figure 1C). The remarkable efficiency of this differentiation system is indicated by the fact that $97 \%$ of the day 3 cells express the SOX17 endodermal marker (Supplemental Figure 1A; supplemental material available online with this article; https://doi.org/10.1172/jci.insight.94954DS1). By day 6, the posterior 
A
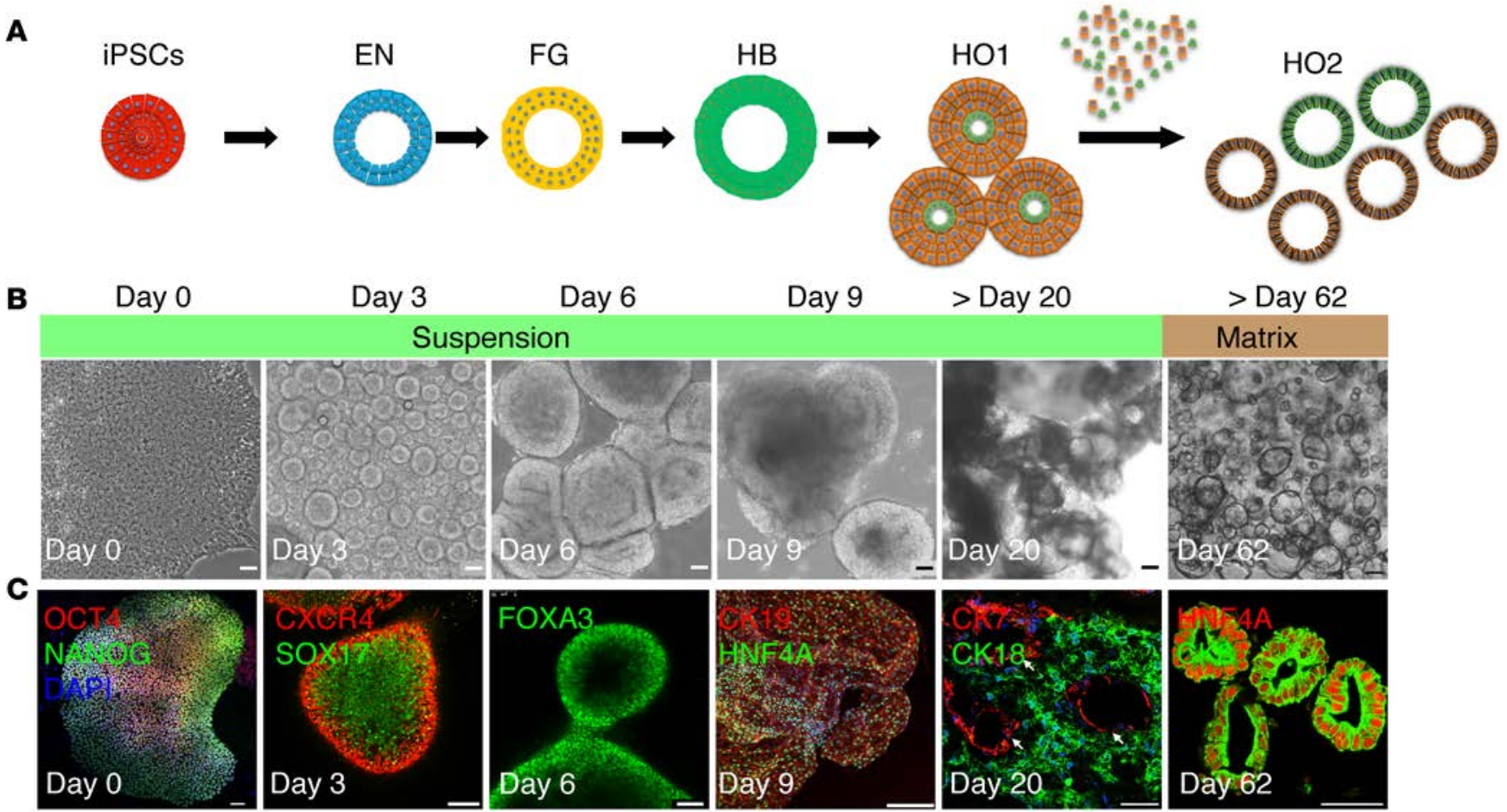

Day 3

Day 6

Day 9

$>$ Day 20

$>$ Day 62

\section{Suspension}
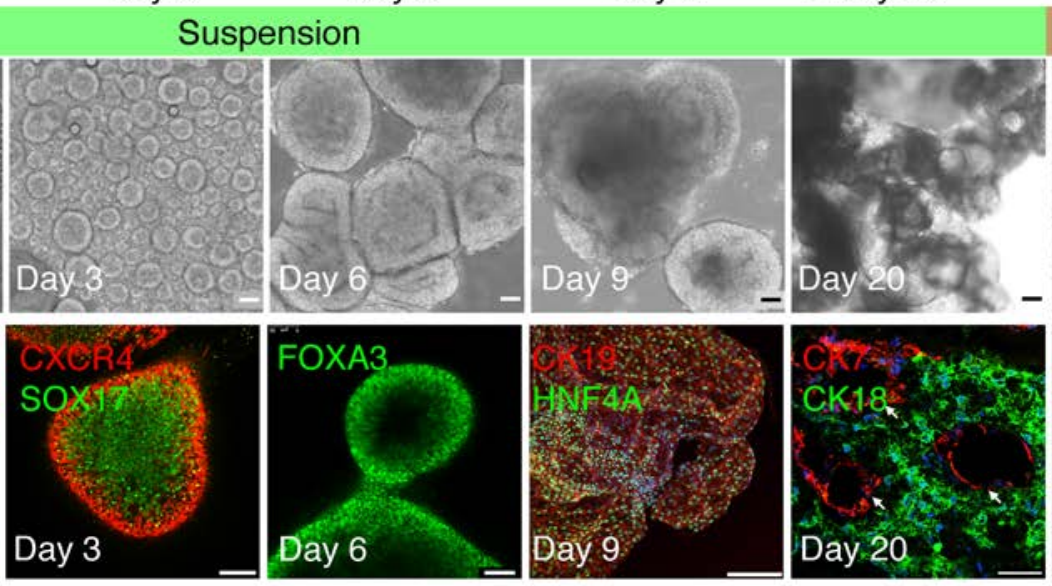

Matrix
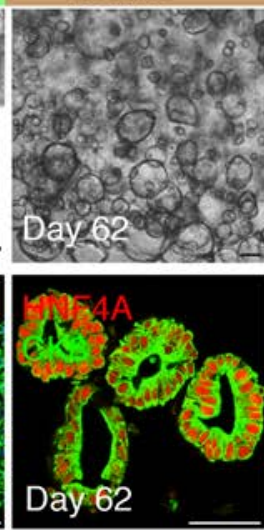

$\mathbf{F}$

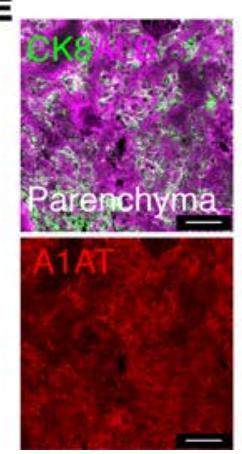

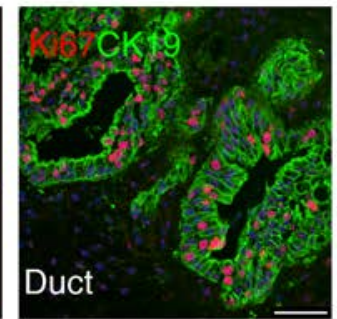

Duct
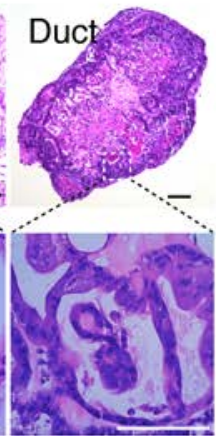

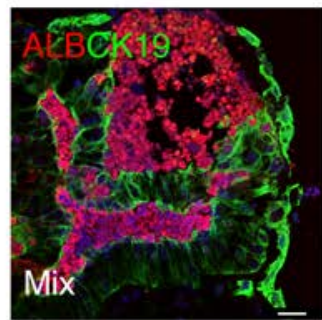

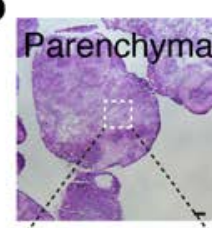
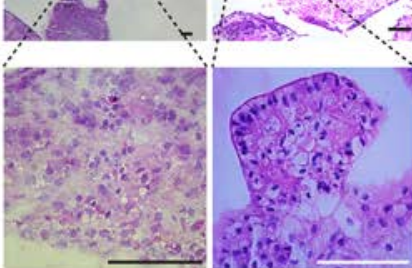

Figure 1. Generation of HOs from iPSCs. (A) A schematic representation of the in vitro culture system and the growth factors used to direct the differentiation of iPSCs into H01s. Then, the cells in a $\mathrm{HO1}$ are dissociated, and (B and C) bright-field and immunostaining images taken on day 0 (iPSCs), day 3 (definitive endoderm, EN), day 6 (foregut, FG), day 9 (hepatoblast, HB), day 20 (H01), and day 62 (HO2) show the morphological and cell marker changes that occur during the development of HOs. The arrows indicate where duct-like structures are located. (D) H\&E staining shows the appearance of 3 different types of HO1. (E and F) Immunostained sections prepared from day 50 parenchymal, ductal, or mixed organoids show the pattern of expression of hepatocyte (ALB, CK8, A1AT), cholangiocyte (CK19), or proliferation (Ki67) markers. Scale bars: $50 \mu \mathrm{m}$.

foregut-like structures express FOXA3 (Figure 1C). By day 9, the developing HOs express multiple markers indicative of hepatoblasts (Figure 1C and Supplemental Figure 1B). For example, 99\% of the day 9 cells expressed a $\mathrm{T}$ box transcription factor (TBX3), which was shown to regulate hepatoblast proliferation and differentiation (34). Also, $63 \%$ of the day 9 cells expressed activated NOTCH1, and similar numbers of cells expressed the Notch ligands JAG1 and DLK1 (Supplemental Figure 1B). After further differentiation, the HO1s expressed the hepatocyte marker CK18 on day 15 (Supplemental Figure 1C). Between days 15 and 20, the $\mathrm{HO} 1$ s formed luminal structures that resemble bile ducts, which are surrounded by $\mathrm{CK} 7^{+}$cells, and HO1 cells reciprocally expressed either a hepatocyte (CK18) or a cholangiocyte marker (CK7) (Figure 1C and Supplemental Figure 1D). Upon closer analysis of H\&E-stained sections of many different organoids, we found that 3 morphologically different types of HO1 were formed: (a) parenchymal organoids where hepatocytes were the predominant cell type; (b) ductal organoids, which had an increased number of ductal structures; and (c) mixed organoids that had both hepatocytes and ductal structures (Figure 1D). Immunostaining confirmed that the hepatocytes in a parenchymal type of HO1 abundantly expressed ALB, CK8, and A1AT (Figure 1E). In ductal and mixed organoids, which were the predominant type of HO1s formed, the 
epithelial cells around the ductal structures abundantly expressed CK19 (Figure 1F). Taken together, these results indicate that the cells in day 9 cultures are bipotent hepatoblasts, which subsequently differentiate into either hepatocytes or cholangiocytes that are present in HO1s. The HO2s also have luminal structures and they express hepatic markers such as HNF4A and CK18 (Figure 1C).

Whole-transcriptome analysis was performed on iPSCs, primary human hepatocytes (PHHs), HO1s, and human liver tissue. For this analysis, over 100 organoids that were generated from a single donor were analyzed. A heatmap, which focused on 2,458 genes that were involved in stem cell differentiation and liver development, was prepared to visualize the relative relationship between the transcriptomes of iPSCs, PHHs, human liver tissue, and HO1s (Supplemental Figure 1E). The dendrogram indicates that HO1s are more closely related to liver tissue than to PHHs; this is because cholangiocytes are present in HO1s and liver, but are absent (or rare) in PHHs. Examination of the genes within this heatmap indicated that there was a lack of expression of pluripotent genes in $\mathrm{HO} 1 \mathrm{~s}$, and that $\mathrm{HO} 1 \mathrm{~s}$ had an increased level of expression of cellular markers for liver lineages and liver development, which included mature hepatocytes, bile duct cells, and NOTCH signaling components. Next, whole-transcriptome analysis was performed to characterize HO2s and their relationship to iPSCs, PHHs, and human liver tissue. This analysis utilized 1,000 HO2s that were prepared from a single donor. A cluster analysis of 1,510 genes indicates that there were major changes in the transcriptome after the cells that were dissociated from an $\mathrm{HO} 1$ were transferred from the growth medium into the differentiation medium. As with the $\mathrm{HO} 1 \mathrm{~s}$, the gene expression profile of $\mathrm{HO} 2 \mathrm{~s}$ resembled that of $\mathrm{PHHs}$ and human liver. Of particular relevance, the level of expression of mRNAs that are of importance for various human liver functions (ADH4, CYP3A4, TTR, GSRA1, TDO2, GSTA1, and FAH) was markedly increased in HO2s (Supplemental Figure 1F).

A serial analysis of the level of mRNA expression for 15 different genes indicates the dynamic changes in gene expression that occur during organoid formation (Supplemental Figure 1G). As examples, SOX17 mRNA expression peaks at day 3; albumin mRNA only appears after day 20 in HO1s and gradually reaches a level that is similar to that in PHHs and liver tissue; TBX3 mRNA appears on day 3, and peaks at the hepatoblast stage; while $A F P$ mRNA appears at the hepatoblast stage, peaks in $\mathrm{HO} 1$ and decreases in $\mathrm{HO} 2$. In contrast, the hepatic lineage marker mRNAs HNF4A, PROX1, and HHEX appear on day 3 and remain elevated through HO2 formation. $C K 19$ and $S O X 9$ mRNA levels, which are important for bile duct morphogenesis, significantly increase at hepatoblast stage, and then peak during HO maturation. Interestingly, $C K 7 \mathrm{mRNA}$ appears later than $C K 19$ mRNA. HNF1b, GGT, and CFTR mRNAs levels increase as the organoids develop, and their expression levels are similar to that of human liver tissue, but these mRNAs are not present in PHHs.

We also performed immunostaining on liver sections at different embryonic stages to assess whether the temporal pattern of antigen expression in developing HOs resembles that of liver during embryonic development. The pattern of expression of HNF4A, TBX3, HNF3 $\beta$, and AFP in day 3 HO cultures was similar to their murine homologs in day 13 (E13) mouse embryonic liver, which is the period when hepatoblasts are formed (35) (Supplemental Figure 1H). The pattern of SOX9 and ECAD expression in E17 liver (which is when bile ducts are present) was similar to that of day $15 \mathrm{HO}$ s, while CFTR expression in E17 liver resembled that of day $30 \mathrm{HO} 1 \mathrm{~s}$ (Supplemental Figure 1I).

HOs have functional properties of liver. Functional assays were performed to determine if HOs had biosynthetic and drug biotransformation properties characteristic of human liver. HOs express the tight junction marker ZO-1 (Figure 2A) that is important for the formation of bile canaliculi. More importantly, those bile ducts formed primary cilia that are similar to those of primary cholangiocytes in vivo (Figure $2 \mathrm{~B}$ ). The organoids had bile acids within their ductules, their cells accumulated glycogen (Figure 2C), and several types of bile acids were abundantly secreted into the supernatant (Figure 2D). A large amount of human albumin $(5-6 \mu \mathrm{g} / \mathrm{ml})$ was produced over a 24 -hour period, which indicated that organoids had substantial biosynthetic capability (Figure 2E). We measured the amount of 2 drug metabolites (M1 and M6), which are generated primarily by a CYP3A4-mediated reaction (36), produced after HOs were incubated with 3.6 $\mu \mathrm{g} / \mathrm{ml}$ clemizole for 24 hours. The amount of M1 and M6 generated by the organoids was 1,033-fold ( $P=$ 0.03 ) and 3,787 -fold $(P=0.007)$, respectively, above that produced by iPSCs. Of note, the amount of the M1 and M6 metabolites produced by HOs after 50 days in culture ( $86 \%$ and $74 \%$, respectively) was close to that produced by PHHs, which were tested after only 24 hours in culture (Figure 2F). After 48-72 hours in culture, PHHs would completely lose their ability to metabolize drugs.

An $\mathrm{HO}$ model for liver regeneration. Liver size increases during the postnatal period due the formation of an increased number of lobules (rather than by an increase in the size of each lobule) (37). Of critical importance to postnatal liver growth, branches must be formed from the existing bile ducts to produce new bile ducts for 
A

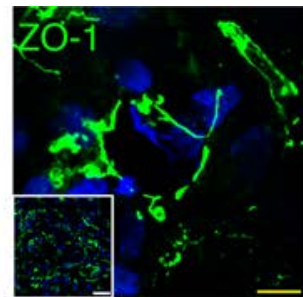

D

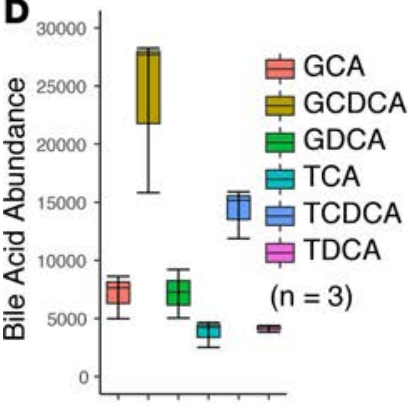

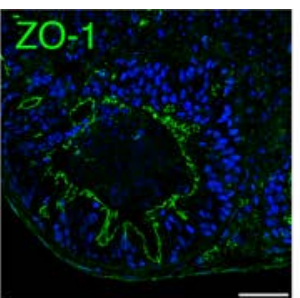

E

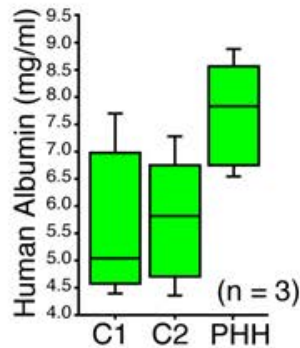

B

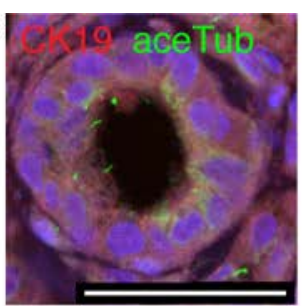

$\mathbf{F}$

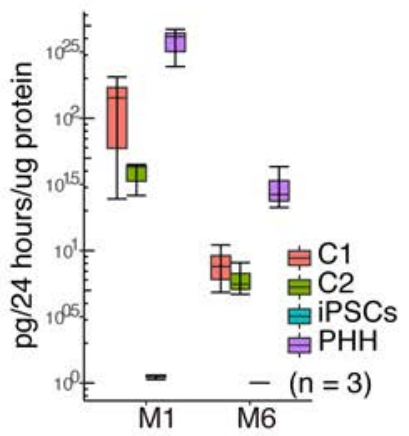

C

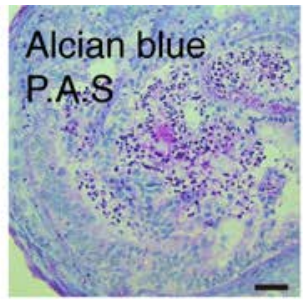

G
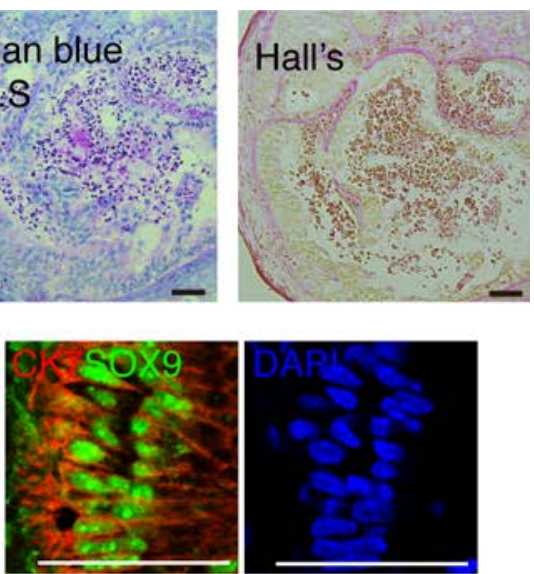

H

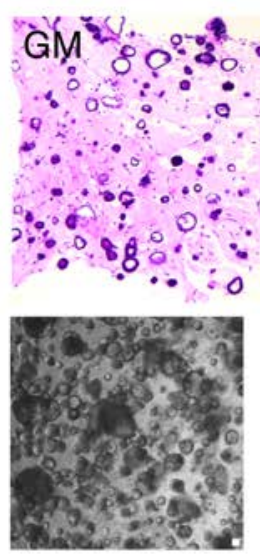

I

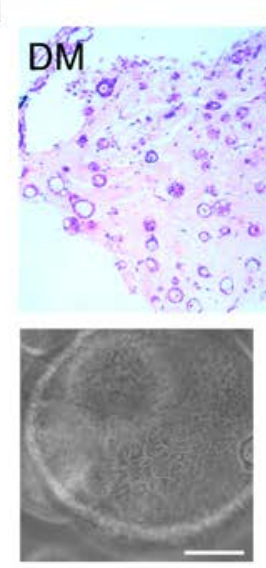

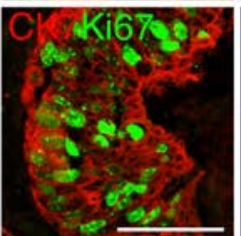

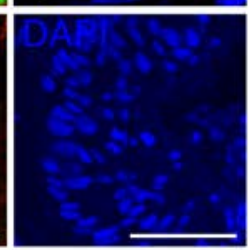

J
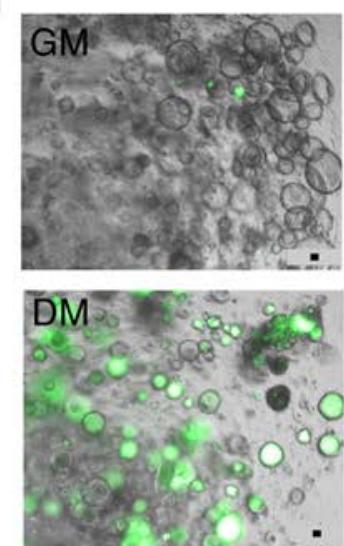

K
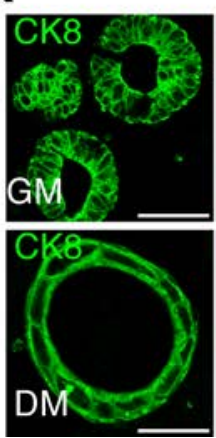
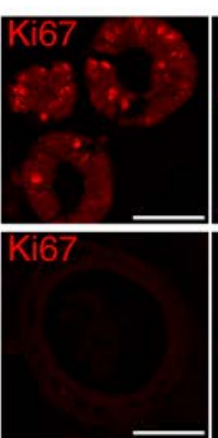

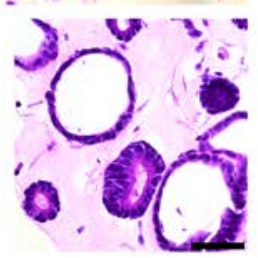

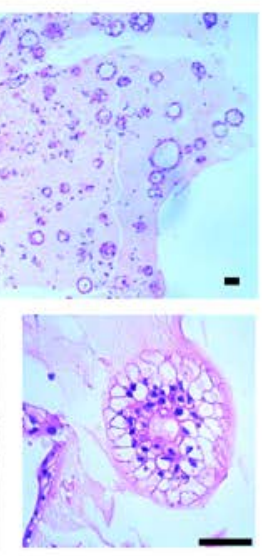

$\mathbf{L}$ 
staining and bright-field images show HO2s on day 6 in growth medium (GM) regenerated from dissociated HO1s (lower and higher magnification) (H), or H02s on day 12 in differentiation medium (DM) (lower and higher magnification) (I). The lower panel is enlarged to show a single H02. (J) Fluorescence and bright-field merged images show that there is abundant bile acid accumulation in the lumen of $\mathrm{HO2s}$ after differentiation. (K and $\mathbf{L}$ ) Confocal images examining the expression of liver lineage markers by HO2s in GM or DM. Scale bars: $50 \mu \mathrm{m}$.

the newly formed lobules (38). To model the processes that could mediate the formation of new lobules and bile duct branching, we determined whether organoids with bile ducts could be formed from the cells that are present in HO1s. We identified in day 50 organoids proliferating cells $\left(\right.$ Ki6 $\left.67^{+}\right)$, which were located in ductules, that coexpressed CK7 and SOX9 (Figure 2G). These SOX9 ${ }^{+} \mathrm{CK} 7^{+}$cells resemble the liver progenitor cells that are found in the ductal plates, which have been shown to regenerate liver under normal physiologic conditions (39). To test the hypothesis that $\mathrm{SOX}^{+} \mathrm{CK} 7^{+}$cells regenerate liver, we then dissociated HO1s into single cells (Supplemental Figure 2A). As expected, those progenitors expanded rapidly in growth medium, which activated Wnt and EGF pathways and inhibited the BMP and TGF pathways (31) (Figure 2H). The dissociated cells from HO1s could be expanded for multiple passages $(>20)$ in the growth medium, and they maintained their ability to form organoids with luminal structures over a 4-month period. To promote further differentiation of HO2s, BMP and NOTCH signaling were activated, and in response to oncostatin $\mathrm{M}$ addition, the HO2s stopped proliferating, and their morphology became more like that of hepatocytes (Figure 2I). At this time, they synthesize lipids and had bile acids (Figure $2 \mathrm{~J}$ ). Immunostaining revealed that $\mathrm{HO} 2$ s prominently express early hepatic lineage markers such as CK8, HNF4A, CK19, EpCAM, and SOX9 in growth medium, which indicates that they resemble hepatic progenitor and/or reactive ductular cells. Also, albumin was only weakly expressed by these cells, and the expression of a mature hepatocyte marker (A1AT) was absent at this stage (Supplemental Figure $2 \mathrm{~A})$. When the cells were grown in a differentiation medium, progenitor marker expression was reduced, and albumin expression was substantially increased (Figure 2, K and L). The cell population within $\mathrm{HO} 2$ cultures consisted of hepatocytes $\left(\mathrm{ALB}^{+} \mathrm{A} 1 \mathrm{AT}^{+} \mathrm{CK} 19-\right)$ and cholangiocytes $\left(\mathrm{CK}^{+} \mathrm{CFTR}{ }^{+}\right)$(Supplemental Figure $\left.2 \mathrm{~A}\right)$. Interestingly, a population of hepatic progenitor cells $\left(\mathrm{SOX}^{+} \mathrm{EpCAM}^{+}\right)$was still present in $\mathrm{HO} 2 \mathrm{~s}$, and $\mathrm{ALB}^{+}$ hepatocytes were located near ductal structures with $\mathrm{CK} 19^{+}$cells (Supplemental Figure 2B).

$J A G 1$ expression in developing organoids. We examined the pattern of JAG1 expression during HO formation. By day 20 , the percentage of $\mathrm{JAG}^{+}$cells in control HOs decreased to $27 \%$ (from $58 \%$ on day 9) (Figure 3A), which could indicate that a subpopulation of $\mathrm{JAG}^{+}$cells in the day 9 (hepatoblast) cultures have differentiated into cholangiocytes. Of note, JAG1 expression was colocalized with a cholangiocyte marker in embryonic mouse liver (Figure 3B). We also investigated whether there was a change in NOTCH signaling components during HO formation. The mRNAs for JAG1 (ligand) and NOTCH2 (receptor) were dramatically increased at the hepatoblast stage. Moreover, the mRNAs for NOTCH transcription factors (HEY1 and HES1) were also increased during HO formation (Figure 3C). Since Notch signaling promotes bile duct formation (40), we examined JAG1 and CK7 expression in control HOs on day 9; this is the time when the hepatoblasts, which will subsequently differentiate into hepatocytes and cholangiocytes, are present. JAG1 and CK7 were expressed at this time, and activated NOTCH1 (NICD) was present in the nucleus (Figure 3D). Since the relative levels of cell surface expression of NOTCH receptors and their ligands on interacting cells affect cell fate determination (41), these changes in the pattern of JAG1 expression may play an important role in bile duct development during $\mathrm{HO}$ formation. It is noteworthy that changes in JAG1 expression are most pronounced in areas where cholangiocytes and bile ducts form. There was also a significant change in the pattern of JAG1 expression in developing $\mathrm{HO}$ cultures. JAG1 ${ }^{+}$cells were initially localized within rosette-like clusters of cells on day 9 , which disappeared as the HOs further differentiated, and $\mathrm{JAG} 1^{+}$cells subsequently appeared in regions near the tubular structures observed on days 15 and 50 (Figure 3E). We also found that there was abundant JAG1 and NICD expression in HO2s that were maintained in growth medium, but their expression was absent when HO2s were in the differentiation medium (Figure 3F). Given their important role in liver development, we investigated whether mesenchymal cells were present in HO cultures. To more fully assess whether mesenchymal cells were present in the organoids, the cells in day 9 , day 20, and day 50 organoids were examined by flow cytometry with an antibody reactive with another mesenchymal cell marker (smooth muscle actin, SMA). The flow cytometry results confirmed that only a small population of cells (1\%-2\%) within the organoids expressed this mesenchymal antigen (Supplemental Figure 2C). Reverse transcription PCR (RT-PCR) analysis of mRNA isolated from these organoids also indicated that mRNAs for SMA and another mesenchymal marker (vimentin) were expressed at detectable lev- 
A

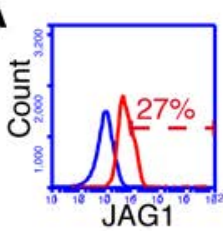

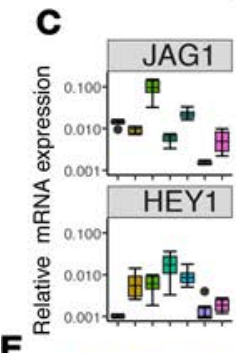

E

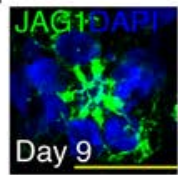

B

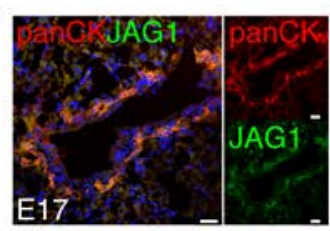

$\mathbf{F}$
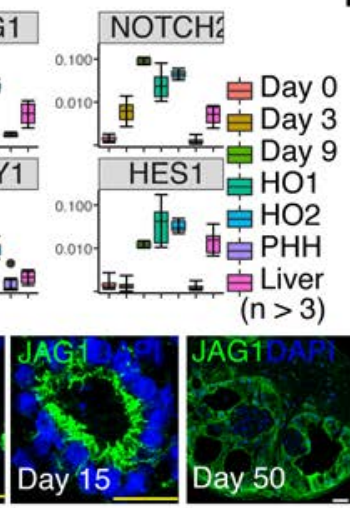

D

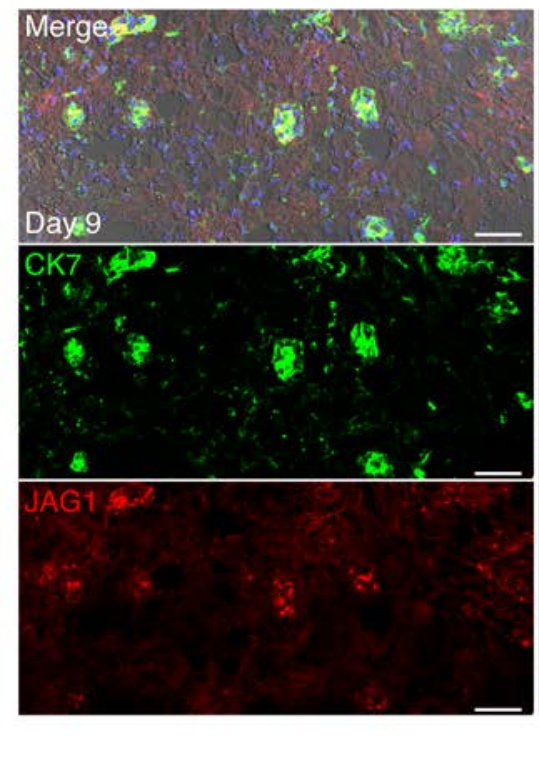

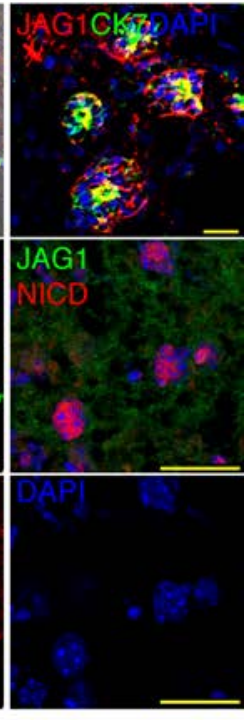

Figure 3. JAG1 expression and NOTCH signaling is altered in ALCS HOs. (A) Flow cytometric analyses showing the proportion of JAC1+ ${ }^{+}$cells present in day 20 control H01s. (B) Immunofluorescence images of E17 liver show that JAG1 expression was colocalized with a cholangiocyte marker (Pan-CK). (C) Quantitative RT-PCR analyses showing the dynamic changes in the expression level for 4 mRNAs in day 0, 3, 9, 20 (HO1), and 62 (HO2) organoid cultures. For comparison, the expression of these 4 mRNAs was also measured in primary human hepatocytes (PHHs) and in human liver tissue. The mRNAs analyzed are involved in NOTCH signaling (VAC1, NOTCH2, HEY1, and HES1). (D) Immunofluorescence images indicate that day 9 control H01s simultaneously express the cholangiocyte marker CK7 and the NOTCH signaling ligand JAG1. There is also evidence of Notch pathway activation, since the intracellular domain of NOTCH1 is detected in these cells (right middle panel). Scale bars: $25 \mu \mathrm{m}$. (E) Immunofluorescence images show the change in the pattern of JAG1 expression from day 9 to day 50 in control H01s. JAG1+ cells were initially localized within rosette-like clusters of cells on day 9 , and then appeared in regions surrounding the developing bile ductal structures. (F) Immunofluorescence images show the change in the pattern of JAG1 and NICD expression in growth media and differentiation media of HO2s. (G) Flow cytometric analyses showing the proportion of CK19+ and JAC1+ cells in control HOs1, $\mathrm{HO2s}$, and PHHs. Scale bars: $50 \mu \mathrm{m}$.

els in the organoids (Supplemental Figure 2D). Immunostaining with a pan fibroblast marker (TE7) indicated that a very small number of mesenchyme-derived cells were present in areas near the tubular duct-like structures in day 50 organoids, and the TE7 $7^{+}$cells also expressed JAG1 (Supplemental Figure 2E). Dual-label flow cytometry was also used to quantitatively characterize the JAG1 ${ }^{+}$cell population in HOs. The cytometry results indicated that $73 \%$ of the cells expressing JAG1 are CK19+ ${ }^{+}$in HO2s, while only $18 \%$ of the JAG $1^{+}$cells in a primary organoid were CK19+ (Figure 3G). Taken together, these results indicate that much of the JAG1 protein in HOs was expressed on cholangiocytes, and many of these are within or near ductular structures. Thus, JAG1-expressing cholangiocytes (and precursor cells), rather than mesenchymal cells, appear to be responsible for mediating the effect of Notch signaling on bile duct formation in organoids. However, since multiple analyses demonstrate that mesenchymal antigens are expressed on some cells within HOs, it is possible that mesenchymal cells could play an important role in organoid development.

Modeling ALGS liver pathology. To determine whether HOs can recapitulate ALGS liver pathology, we first examined liver tissue prepared from 3 subjects with ALGS liver disease. Their liver tissue had typical features of ALGS liver disease, which included a paucity of bile ducts, intrahepatic cholestasis, and fibrosis (Figure 4A). The level of expression of mRNAs for several NOTCH signaling components (JAG1, NOTCH2, HEY1) and cholangiocyte markers (CK7, CFTR) was dramatically decreased in ALGS liver tissue relative to normal liver tissue (Figure $4 \mathrm{~B}$ ). The mRNA data demonstrate that there is evidence of impaired NOTCH signaling in human ALGS liver tissue.

We prepared iPSCs from fibroblasts obtained from 3 controls (C1, C2, and C3), and from 2 ALGS subjects (ALGS1 and ALGS2) with liver defects that were severe enough to require a liver transplant (Supplemental Figures 3 and 4). ALGS1 had a heterozygous mutation that introduced a termination codon (C829X) into the JAG1 coding sequence (Figure 4C), while ALGS2 had a splice site mutation that altered JAG1 transcript splicing (Supplemental Figure 3). We compared the properties of HOs prepared from ALGS1 and ALGS2 iPSCs with those of control organoids. Control iPSCs formed intact 
A
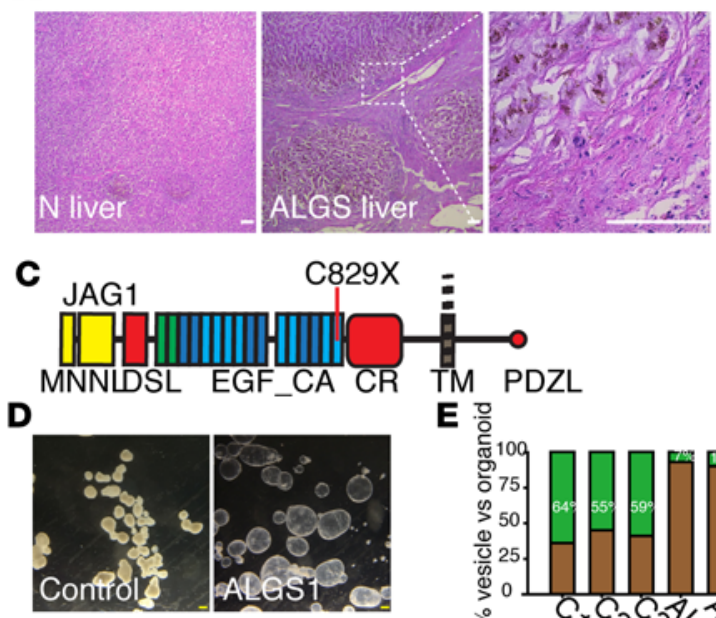

F

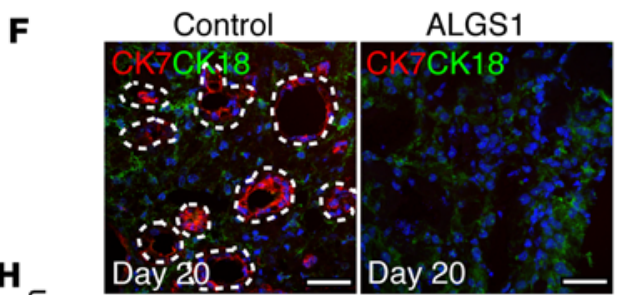

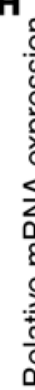

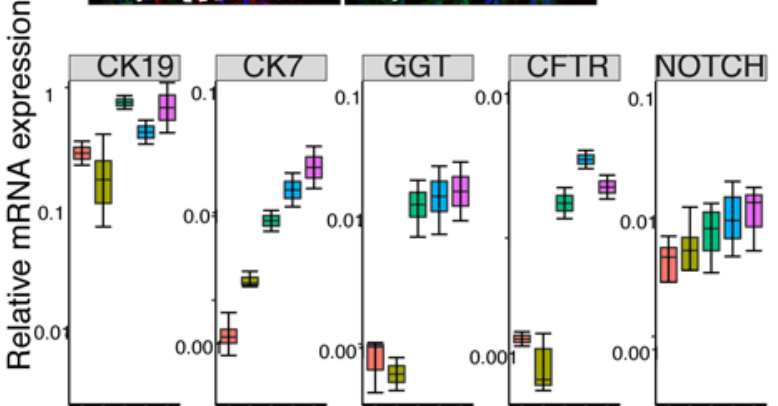

I

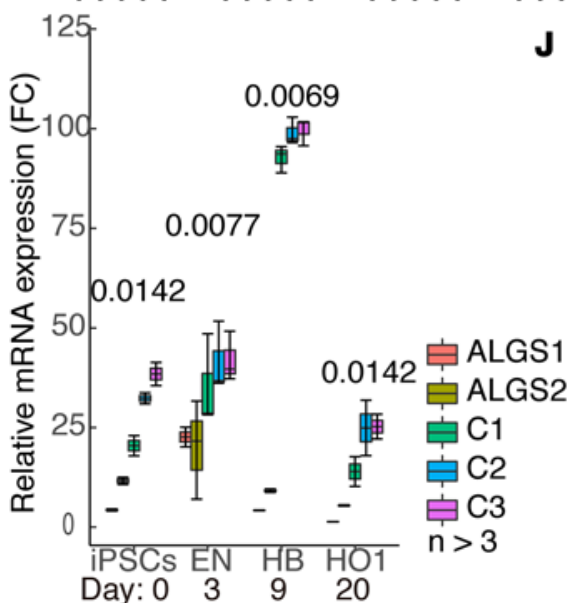

$E$ :응

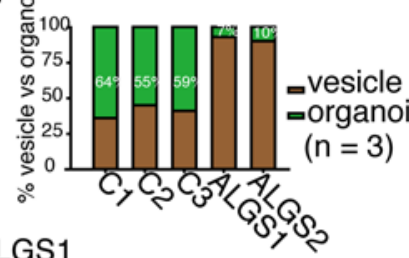

B
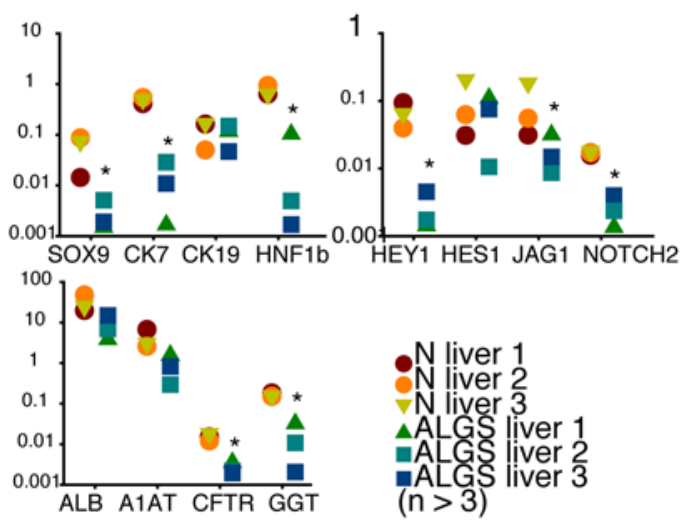

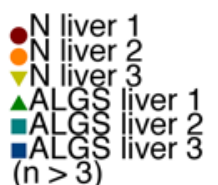

G
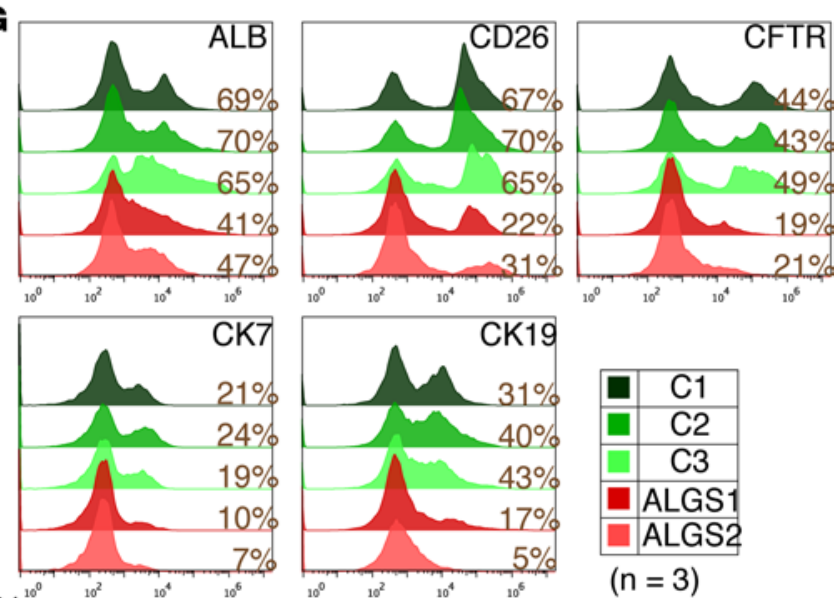

泉ALGS1
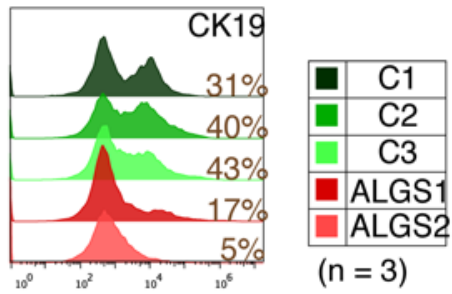

$(n=3)$

Figure 4. HOs recapitulate the impaired bile duct formation that is characteristic of ALCS liver pathology. (A) H\&E-stained sections of normal (N) and ALCS liver tissue. The boxed area within the ALCS liver section is enlarged in the right panel to show an area with cholestasis and fibrosis. Scale bars: 100 $\mu \mathrm{m}$. (B) Quantitative RT-PCR analyses for mRNAs encoding cholangiocyte differentiation (SOX9, CK7, CK19, and CFTR), hepatocyte (ALB, A1At, GGT, and HNF1b), and NOTCH signaling markers (HEY1, HES1, JAG1, and NOTCH2) in normal and ALGS liver tissues. ${ }^{*} P<0.05$ for comparison of the gene expression level (2^-ct) in normal versus ALGS liver tissue. (C) A schematic representation of the C829X mutation site (ALGS1) within an EGF-like domain of the JAG1 protein. (D) Bright-field images of ALGS and control HO1 cultures on day 30. While control iPSCs form intact H01s, ALGS iPSCs formed large (>2 mm) fluid-filled vesicles. Scale bars: $1 \mathrm{~mm}$. (E) The number of vesicles and organoids formed in day $30 \mathrm{HO1}$ cultures generated from 3 control or 2 ALCS iPSC lines are shown. Each bar is the average of 3 independent determinations, and each culture had more than 100 evaluable structures. (F) Immunofluorescence images showing CK7 and CK18 expression in control and ALGS H01s. (G) Flow cytometric analyses showing the proportion of cells expressing hepatocyte 
(ALB) or cholangiocyte (CD26, CK7, CK19, and CFTR) markers in day 20 H01s prepared from control and ALGS iPSCs. (H) Quantitative RT-PCR analyses for CK19, CK7, GCT, CFTR, and NOTCH2 mRNA expression in day 20 control and ALGS organoids. (I) Quantitative RT-PCR analyses for JAG1 mRNA expression in iPSCs, endodermal spheres, hepatoblasts, and H01s formed from 3 control and 2 ALCS individuals. The actual $P$ values for the difference in expression between control and ALGS cells are indicated at each comparison. FC, fold change. (J) Immunofluorescence images indicate that JAG1 is expressed in the cells surrounding the lumen of control H01s, while JAG1 expression is reduced in ALGS1 H01s. Representative images from $n=6$ technical replicates are shown. (K) Bright-field images of H02s formed 12 days after control or ALGS organoids were dissociated. The number of intact HO2s formed within these cultures is shown. The results are normalized relative to 1,000 cells plated. Scale bars: $50 \mu \mathrm{m}$ (J and $\mathbf{K}$ ).

HO1s with ductular structures. While ALGS HO1s had CK18 ${ }^{+}$cells, they did not form apparent tubular structures, nor did they have many $\mathrm{CK} 7^{+}$cells (Figure $4 \mathrm{~F}$ ). A subpopulation analysis by flow cytometry indicated that ALGS HO1s had somewhat fewer albumin ${ }^{+}$hepatocytes than control HOs, but there was a more marked reduction in $\mathrm{CD}_{2} 6^{+}, \mathrm{CK}^{+}, \mathrm{CK}_{1} 9^{+}$, and $\mathrm{CFTR}^{+}$cholangiocytes (42) in ALGS HO1s (Figure 4G). The efficiency of organoid formation by control and ALGS iPSCs was dramatically different (Figure 4E). While over $50 \%$ of the structures formed in control HO1 cultures were intact organoids, over $90 \%$ of the structures formed in ALGS cultures were vesicles (Supplemental Figure 5A). We examined the intact vesicles formed in the ALGS organoid cultures, and found that the fluid-filled vesicles (Figure 4D) were lined by hepatocyte-like cells (Supplemental Figure 5B).

We also compared the expression of mRNAs for bile duct differentiation markers (CK19, CK7, GGT, and CFTR) and NOTCH2 in control and ALGS HO1s. Consistent with results obtained with ALGS and control liver tissues, the ALGS organoids had markedly reduced levels of $C K 7, G G T, C F T R$, and NOTCH2 mRNAs relative to control organoids (Figure 4H). Since CK19 is expressed on progenitor cells, CK19 mRNA levels in ALGS organoids was only slightly reduced relative to control organoids. The level of expression of mRNAs encoding $2 \mathrm{NOTCH}$ downstream target genes (HEY1 and HES1), which are involved in bile duct formation (43), were also significantly decreased in day 20 ALGS HO1s (Supplemental Figure 5C). In contrast, there was no significant difference in the level of expression of other mRNAs (HNF4A and $\alpha$-fetoprotein) between control and ALGS HO1s at the hepatoblast stage (Supplemental Figure 5D). We examined the level of expression of mRNAs for other hepatocyte and cholangiocyte markers in day 20 control and ALGS HO1s. In contrast to the HNF4A and $\alpha$-fetoprotein mRNAs, the levels of expression of certain hepatocyte (HHEX, albumin, and $A A T)$ and cholangiocyte (HNF1B and $S O X 9$ ) markers in ALGS HOs were significantly reduced relative to control HO1s (Supplemental Figure 5E). Since the JAG1 mutation is causative for ALGS, we compared the pattern of JAG1 mRNA and protein expression in control and ALGS HO1 cultures. JAG1 mRNA underwent dynamic changes in expression in control HO1 cultures, and JAG1 mRNA expression peaked during the hepatoblast stage (day 9). However, JAG1 mRNA levels were significantly decreased during all developmental stages in ALGS HO1s, and did not increase during the hepatoblast stage (Figure 4I). Furthermore, immunostaining revealed that $\mathrm{JAG}^{+}$cells surrounded the luminal structures formed by control HOs, but JAG1 expression was reduced in day 9 ALGS HOs (Figure 4J). The lack of increased JAG1 expression during a period that is critical for bile duct development may explain the paucity of bile ducts found in ALGS HO1s. Given the reduction in cholangiocytes and in duct-like structures in ALGS HO1s, we examined their ability to mediate the intraluminal transport of a dye (rhodamine 123), which is used to assess multidrug resistance protein-1-dependent (MDR1-dependent) transport by normal cholangiocytes (44). Control HO1s could transport rhodamine 123 into their intraluminal space, while ALGS HO1s could not (Supplemental Figure 5, F-H). Hence, ALGS HO1s had a diminished ability to form duct-like structures, to mediate cholangiocyte-dependent biliary transport, and to produce intact organoids. Next, we compared the regenerative capacity of ALGS organoids. Control HO2s could be expanded for several passages in the growth medium. In contrast, HO2s could not be generated from ALGS-derived HO1s (Figure $4 \mathrm{~K}$ ). Thus, control HO1s have progenitor cells with regenerative potential, which are critical for postnatal liver development. In contrast, in addition to their reduced ability to form bile ducts, ALGS organoids have impaired regenerative ability.

Genome engineering: ALGS pathology is dependent upon the ALGS mutation. We combined CRISPR-associated protein 9 (Cas9) genome editing with the piggyBac transposon system to introduce and revert a disease-causing mutation (C829X [ALGS1]) in control and ALGS iPSCs. The strategy for introducing or reverting the mutations, for selecting cells with desired alterations, and for characterizing their genomic changes is fully described in the supplemental information (Supplemental Figure 6A). Using these methods, the ALGS1 (C829X) mutation was introduced into iPSCs generated from 2 different control individuals ( $\mathrm{C} 1$ and $\mathrm{C} 2)$ to 
A

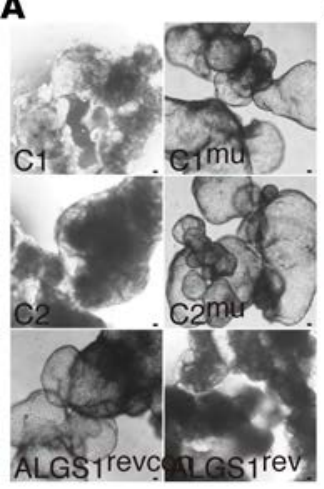

B

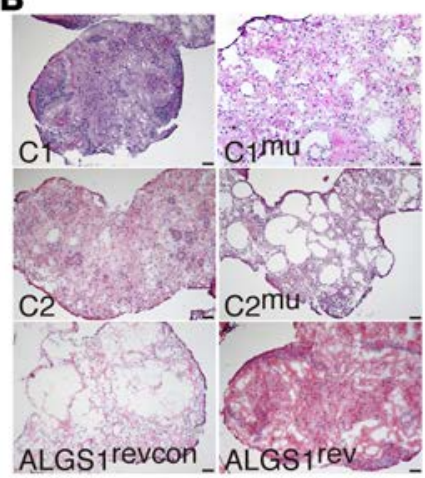

C
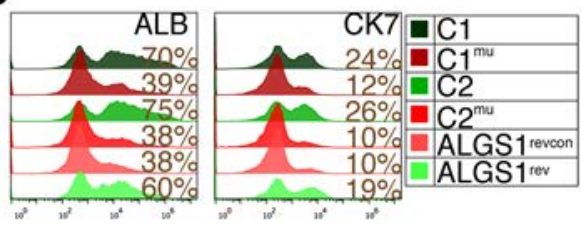

D

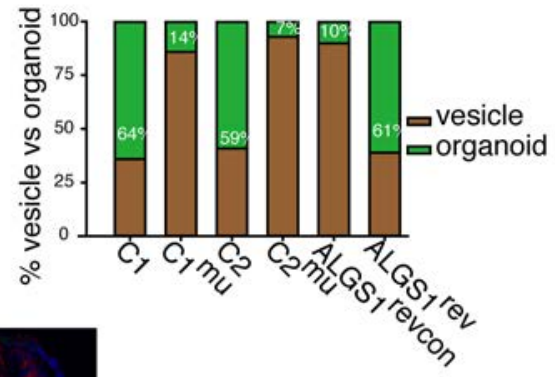

H

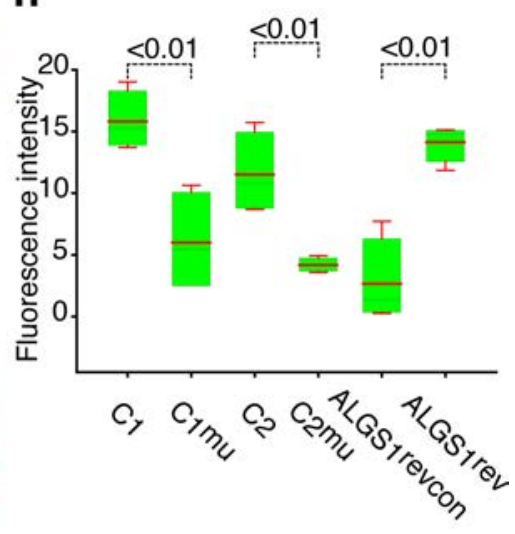

Figure 5. Characterization of iPSC lines with a CRISPR-engineered ALGS1 mutation (C829X). (A and B) Bright-field images and H\&E-stained cryosections of day $25 \mathrm{HO1}$ cultures. The cultures were generated from 2 control $(C 1, C 2)$ iPSC lines prepared and from the following CRISPR-engineered iPSC: C1 ${ }^{\mathrm{mu}}$ and C2 ${ }^{\mathrm{mu}}$, which have the heterozygous C829X mutation; ALGS1 ${ }^{\text {rev }}$, where the ALGS1 mutation was reverted to wild type; and ALGS1revcon, which has the ALGS1 mutation but is a control for the genome engineering process. (C) Flow cytometric analyses showing the proportion of cells expressing hepatocyte (ALB) or cholangiocyte (CD26, CK7, CK19, and CFTR) markers in day $20 \mathrm{HO1s}$ prepared from C1, C1 ${ }^{\mathrm{mu}}, \mathrm{C2}, \mathrm{C2}{ }^{\mathrm{mu}}$, ALGS1 ${ }^{\text {rev, }}$, and ALGS revcon iPSC lines. (D) The JAG1 allele determines the efficiency of formation of intact H01s. The number of fluid-filled vesicles and intact organoids in $\mathrm{HO1}$ cultures generated from the indicated iPSC lines was measured on day 30. The proportion of fluid-filled vesicles formed by iPSCs with a mutated JAG1 (C1mu, C2 ${ }^{\text {mu }}$, ALGS revcon) was much greater than by those with an un-mutated JAG1 (C1, C2, ALGS1 ${ }^{\text {rev }}$. Each bar is the average of 3 independent determinations, and each culture had more than 100 evaluable structures. (E and F) Immunofluorescence staining of the H01 cultures with anti-human albumin, anti-human CK8, and anti-human CK7 antibodies. The sections were also stained with DAPI to indicate the nuclei. Irrespective of whether they had a normal or mutated JAG1 allele, all H01s expressed albumin and CK8. However, CK $7^{+}$cholangiocytes are only abundant in H01s prepared from lines (C1, C2 or ALGS1 $1^{\text {rev}) ~ w i t h ~ a ~ w i l d-t y p e ~ J A G 1 . ~ E a c h ~}$ analysis was reproduced in 3 independent experiments. (C) Quantitative RT-PCR analysis of JAG1 mRNA expression in day 9 H01s prepared from different iPSCs. Each bar is the average of 3 independent cultures, and the results were normalized relative to control iPSCs. JAG1 mRNA levels were 10-fold lower in $\mathrm{C1}^{\mathrm{mu}}, \mathrm{C2}^{\mathrm{mu}}$, and ALGS1 ${ }^{\text {revcon }} \mathrm{HO1s}$ relative to wild-type H01s $(P<0.01)$, but unaltered in ALGS1 ${ }^{\text {revcon }} \mathrm{iPSCS}(P>0.05)$. FC, fold change. (H) The fluorescence intensity within the lumina of the indicated $\mathrm{HO1}$ was normalized relative to the area. The red line is the average of 3 independent measurements, and the box indicates the first and third quartiles. All H01s with a JAG1 mutation were significantly impaired in their ability to transport rhodamine 123 into the ductal lumen. Scale bars: $50 \mu \mathrm{m}$ (all panels).

produce the $\mathrm{C}^{m u}$ and $\mathrm{C} 2^{m u}$ lines, and the JAG1 mutation in ALGS1 iPSCs was reverted to wild type in the ALGS1 ${ }^{\text {rev }}$ line (Supplemental Figure 6B and Supplemental Figure 7). The ALGS1 ${ }^{\text {revon }}$ iPSC line was produced as a control for the off-target effects of the genome engineering process; the piggyBac transposon was inserted and removed from ALGS1 iPSCs, but clones were selected that retained the heterozygous ALGS1 mutation.

The mutated and reverted iPSCs were induced to form HOs, and the morphology of HOs with engineered JAG1 mutations $\left(\mathrm{C}^{m u}\right.$ and $\mathrm{C}^{m u}$ ) was significantly altered (Figure 5, A and $\mathrm{B}$ ). The $\mathrm{C}^{m u}$ and $\mathrm{C}^{m u}$ 
A

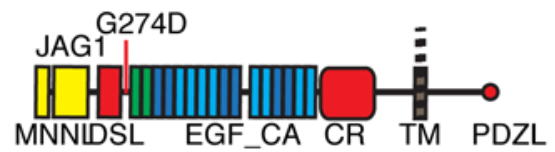

B

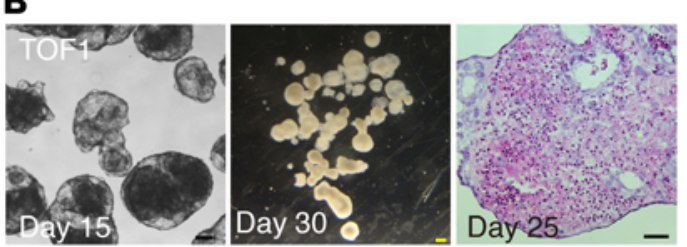

C

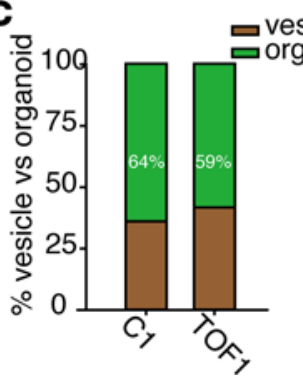

E

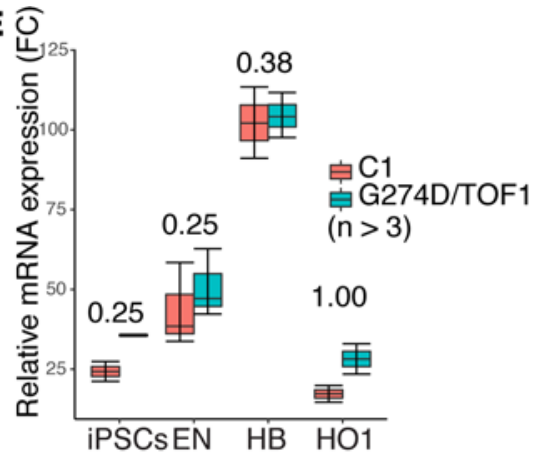

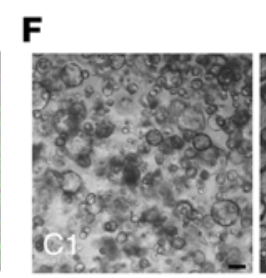
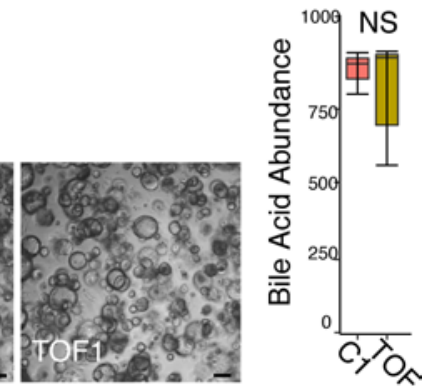

Figure 6. The G274D JAG1 mutation does not alter H01 properties. (A) A schematic representation of G274D mutation (TOF1), which is located within the EGF-like domain of the JAG1 protein. (B) Bright-field images of day 15 and day 30 H01s prepared from iPSCs with a heterozygous TOF1 mutation. Right image: H\&E-stained cryosection of a day 25 TOF1 organoid. These images indicate that TOF1 organoids have a morphology that is similar to that of control organoids. Scale bars: $1 \mathrm{~mm}$. (C) The number of fluid-filled vesicles and of intact organoids formed on day 30 by H01s generated from the control (C1) or TOF1 iPSC lines. (D) Immunofluorescence staining of a day 50 TOF1 HO1 with albumin, CK19, and SOX9 antibodies. (E) Quantitative RT-PCR analyses of JAG1 mRNA expression in iPSCs, endodermal spheres (day 3), hepatoblasts (day 9), and H01s (day 20) formed from C1 and TOF1 iPSCs. Each measurement is the average of 3 independent determinations. FC, fold change. (F) Bright-field images showing the HO2s formed on day 12 by the cells obtained after dissociation of the control (C1) and TOF1 H01s. The number of HO2s formed in the day 12 cultures was measured, and each bar is the average of 3 independent determinations. Scale bars: $50 \mu \mathrm{m}$ (D and $\mathbf{F})$.

HOs also had very similar alterations in their morphology. The $\mathrm{C}^{m u}$ and $\mathrm{C} 2^{m u}$ organoids had less refractivity, and relative to the compact and well-organized structures formed by control organoids, there was an abundance of acellular cysts formed in the $\mathrm{C}^{m u}$ and $\mathrm{C}^{m u}$ cultures. After reversion of the ALGS1 mutation $\left(A L G S{ }^{r e v}\right)$, the HOs recovered their capacity to form well-organized structures with bile ducts. A flow cytometric analysis indicated that the relative numbers of albumin ${ }^{+}$hepatocytes and $\mathrm{CK} 7^{+}$cholangiocytes in HOs with an engineered ALGS1 mutation (C829X) were decreased relative to control HOs. However, when this ALGS mutation was reverted to wild type, the organoids had a marked increase in hepatocytes and cholangiocytes (Figure 5C). The efficiency of intact organoid formation was also determined by the JAG1 allele (Figure 5D). While approximately $60 \%$ of the structures formed by $\mathrm{C} 1, \mathrm{C} 2$, and ALGS ${ }^{\text {rev }}$ cultures on day 20 were intact organoids, only $7 \%-14 \%$ of those formed in $C 1^{m u}, C 2^{m u}$, and ALGS1 revoon cultures on day 20 were intact organoids. We also used immunostaining to examine marker expression in these HOs. $\mathrm{C}^{m u}$ and $\mathrm{C2}^{m u} \mathrm{HOs}$ had cells expressing hepatocyte $\left(\mathrm{ALB}^{+} \mathrm{CK} 8^{+}\right)$markers, but they had markedly reduced levels of $\mathrm{CK} 7^{+}$cholangiocytes, and they did not form bile duct-like structures surrounded by $\mathrm{CK} 7^{+}$cells (Figure 5 , $\mathrm{E}$ and $\mathrm{F})$. However, ALGS1 ${ }^{\text {rev }}$ organoids completely recovered their ability to form bile duct-like structures, and had $\mathrm{CK} 7^{+}$cells. The morphology of $\mathrm{ALGS1}{ }^{\text {revon }}$ organoids, which serve as a control for the engineering process, resembled that of the $\mathrm{C} 1^{m u}$ and $\mathrm{C} 2^{m u} \mathrm{HOs}$.

We also examined JAG1 mRNA expression in the HO cultures at the hepatoblast stage (day 9). JAG1 mRNA expression levels were reduced 10-fold in the day $9 \mathrm{C1}^{\mathrm{mu}}, \mathrm{C} 2^{m u}$, and ALGS1 ${ }^{\text {revon }}$ cultures relative to control HOs, while JAG1 mRNA levels in ALGS1 ${ }^{\text {rev }} \mathrm{HOs}$ were equivalent to control HO cultures (Figure 5G). $\mathrm{C1}^{m u}, \mathrm{C}^{m u}$, and $\mathrm{ALGS} 1^{\text {revon }}$ HOs could not transport rhodamine 123 into their lumen, while $\mathrm{C} 1, \mathrm{C} 2$, and ALGS1 ${ }^{\text {rev }} \mathrm{HOs}$ could do this (Figure $5 \mathrm{H}$ and Supplemental Figure 8). These results indicate that in $\mathrm{HO}$ cultures, the ability to form duct-like structures with cholangiocytes, to develop dynamic changes in JAG1 mRNA expression, biliary transport function and the efficiency of organoid formation are all determined by the presence or absence of the $J A G 1$ mutation. 
A

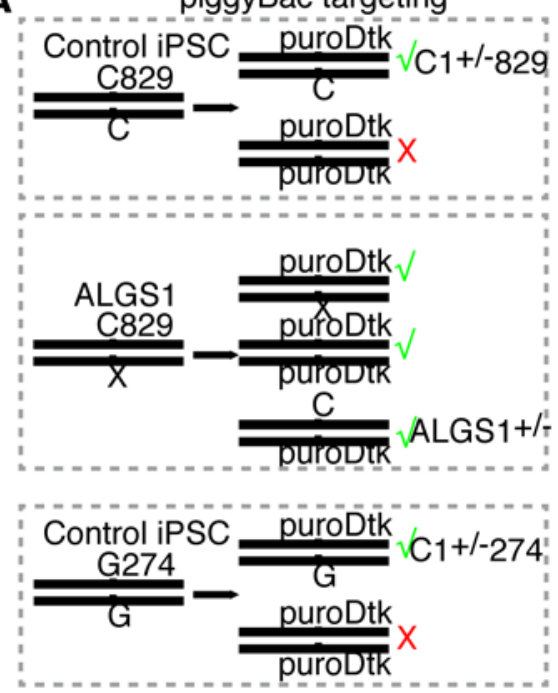

E

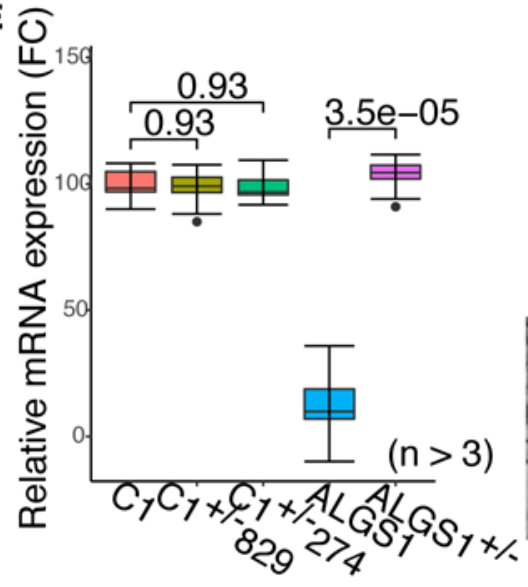

B

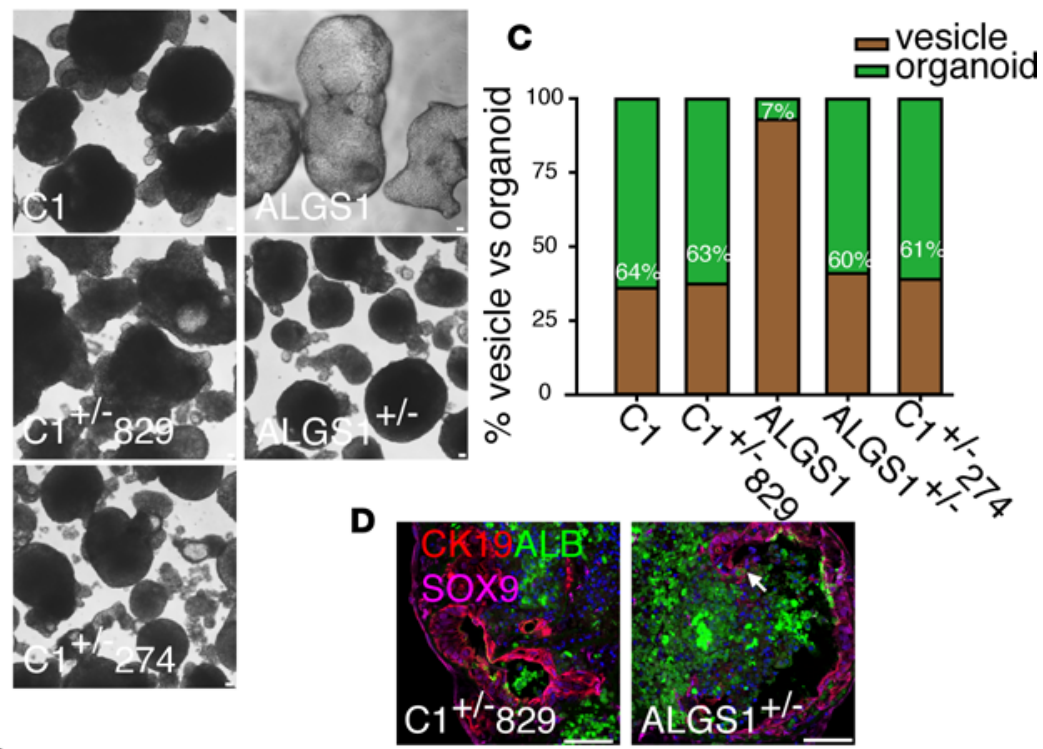

$\mathbf{F}$
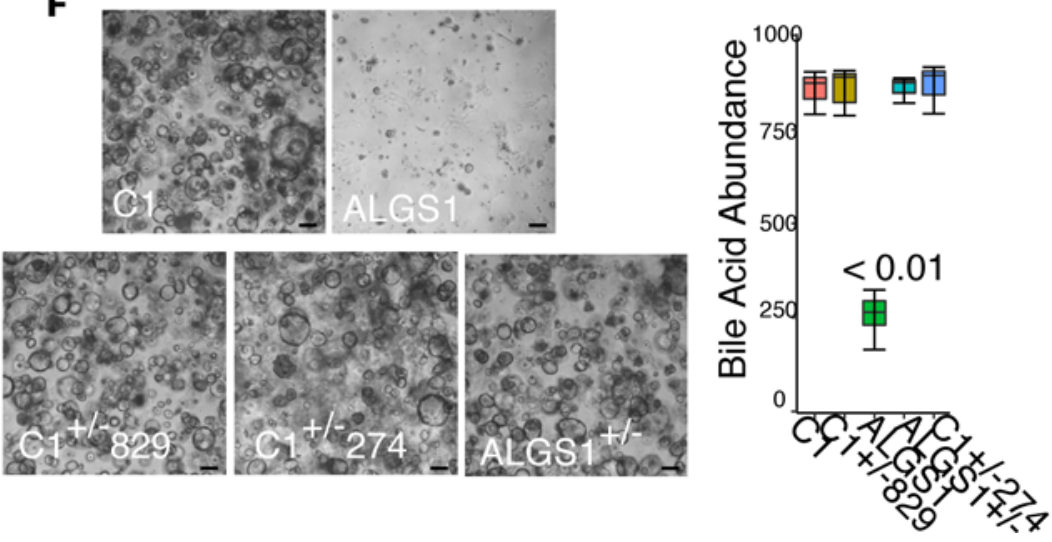

Figure 7. The JAG1 mutation has a dominant-negative effect. (A) The strategy for producing iPSC lines with a heterozygous JAG1 knockout. The 3,079-bp puro $\Delta$ tk cassette was inserted at the JAG1 C829 site in control (C1) or ALGS1 iPSC lines by CRISPR-mediated genomic engineering. After drug treatment, iPSC lines with a heterozygous JAG1 knockout ( $C 1^{+/-} 829$ and ALGS1+/-) were selected for further study. The cassette was also inserted at the JAG1 C274 site in control (C1) iPSCs to produce the C1+/-274 iPSC line. (B and C) Bright-field images of day 25 H01s generated from the indicated iPSC line, and the number of vesicles and organoids formed in the H01s generated from the indicated iPSC lines are shown. Of note, all iPSC lines with a heterozygous JAC1 knockout $\left(\mathrm{C1}^{+1-} 829, \mathrm{C1}^{+/-} 274\right.$, and $\left.\mathrm{ALCS1}{ }^{+-}\right)$could form liver organoids as efficiently as the control (C1) iPSC line. Each bar is the average of 3 independent determinations, and each culture had more than 100 evaluable structures. (D) Immunofluorescence staining of day 50 organoids produced from 2 different iPSC lines with JAG1 heterozygous knockouts (ALCS1+/- and C1+/-829). The albumin ${ }^{+}$and CK19+ cells are clearly seen in the $\mathrm{C}^{+/-} 829$ organoids, and the arrow indicates the location of $C K 19^{+}$cells in an ALGS1+/- organoid. (E) RT-PCR analysis of JAG1 mRNA expression in day 9 H01s prepared from the indicated iPSC lines. Of note, the JAG1 mRNA levels in all of the JAG1 heterozygous organoids was equivalent to that of the control (C1) organoid, and was markedly increased relative to that in the ALGS1 organoid. Each bar is the average of 3 independent determinations. FC, fold change.(F) Bright-field images showing H02s formed from the cells obtained after dissociation of C1, C1 ${ }^{+/-} 829$, ALGS1, ALGS1+/-, and $\mathrm{C1}^{+/-} 274 \mathrm{HO1s}$. The images shown were prepared on day $12 \mathrm{HO} 2 \mathrm{~s}$. Scale bars: $50 \mu \mathrm{m}$ (all panels). The number of H02s formed in the day 12 cultures was quantified, and each bar is the average of 3 independent determinations. While the formation of HO2s was impaired in the presence of the ALGS1 mutation, the cells in all organoids with heterozygous JAG1 mutations (C1+/-829, ALGS1+/-, and C1+/-274) could efficiently form HO2s.

The JAG1 mutation determines the liver phenotype. Thirteen individuals within a large kindred segregating autosomal dominant TOF were found to have a heterozygous missense (Gly274Asp) mutation in the second EGF domain of JAG1 (45) (Figure 6A). Although these individuals had the classic cardiovascular features of TOF, they did not have any liver or bile duct abnormalities. The Asp274-mutated protein was shown to have an altered glycosylation pattern, which reduced its cell surface expression and ability to activate the Notch pathway (29). To investigate whether the type of JAG1 mutation directly affected the liver phenotype, the Gly274Asp JAG1 mutation was engineered into an iPSC line to produce the TOF1-iPSC line (Supplemental Figure 9). Of note, the C829X JAG1 mutation dramatically altered the ability of the same parental iPSC line 
to form liver organoids. However, when TOF1 iPSCs were induced to form HOs, the resulting organoids had the same morphology and had the same efficiency of formation of intact organoids as control iPSCs (Figure 6, B and C). Just as in control organoids, TOF1 HOs had luminal structures that were surrounded by CK19+ cells (Figure 6D); JAG1 mRNA underwent dynamic changes in expression in developing TOF1 HO cultures, which peaked during the hepatoblast stage (Figure 6E); and dissociated cells from TOF1 organoids efficiently regenerated secondary organoids (Figure $6 \mathrm{~F}$ ). In contrast to the dramatic effects caused by the C829X or ALGS2 mutations, the G274D mutation did not alter the pattern of JAG1 mRNA expression, bile duct formation, or organoid formation/regeneration in $\mathrm{HO}$ cultures.

Is ALGS liver disease caused by JAG1 haploinsufficiency? Our liver organoids and genome engineering method were also used to investigate whether ALGS liver pathology results from $J A G 1$ haploinsufficiency. During our genome engineering process using the piggyBac transposon, there is a step where a 3,079-bp cassette (puro $\Delta$ tk) is inserted at a desired site, which enables drug selection to be used to obtain iPSCs with desired mutations. The insert is removed by transposase expression in a subsequent step to create a seamless genome modification. However, since cassette insertion disrupts the expression of one JAG1 allele, it creates an iPSC with a heterozygous JAG1 knockout. By retaining iPSCs with the insert (prior to transposase expression), we generated several iPSC lines with an insertion-mediated heterozygous $J A G 1$ knockout. We prepared control $\left(\mathrm{C}^{+/-} 829\right)$ and ALGS1 (ALGS1 ${ }^{+/}$) iPSCs with heterozygous $J A G 1$ knockout, because the puro $\triangle \mathrm{tk}$ was inserted at the C829 position within $J A G 1$. Irrespective of their original genotype, these cell lines had a heterozygous JAG1 knockout (Figure 7A and Supplemental Figure 10). Unlike iPSCs with a heterozygous ALGS mutation (ALGS1 and ALGS2) that could not efficiently form organoids, the iPSC lines $\left(\mathrm{C}^{+/-} 829\right.$ and $\left.\mathrm{ALGS} 1^{+/-}\right)$with a heterozygous $J A G 1$ knockout were able to efficiently form intact liver organoids (Figure 7, B and C). Over 60\% of the structures formed by control or heterozygous knockout iPSCs were organoids, while less than $10 \%$ of the structures formed by ALGS1 iPSCs were intact organoids. The puro $\Delta$ tk cassette was also inserted at the G274 locus to produce another iPSC line $\left(\mathrm{C}^{+/-274}\right)$ with a heterozygous $J A G 1$ knockout. The $\mathrm{C}^{+/-} 274$ iPSC line was also able to efficiently form intact liver organoids. Similar to control organoids, the heterozygous knockout lines formed HOs with luminal structures that were surrounded by CK19+ cells (Figure 7D), and they had a high level of JAG1 mRNA expression during the hepatoblast stage (Figure 7E). Also, the loss of dynamic changes in $J A G 1$ mRNA expression, which occurred during the formation of ALGS1 organoids, was rescued after the ALGS1 mutation was converted to a heterozygous knockout (ALGS1 $\left.{ }^{+/}\right)$. Moreover, the cells that were dissociated from $\mathrm{ALGS}^{+/-}, \mathrm{Cl}^{+/-} 829$, and $\mathrm{C1}^{+/-} 274$ organoids could form secondary organoids as efficiently as those of control organoids (Figure 7F). These results clearly indicate that ALGS liver abnormalities are caused by a dominant-negative effect of the ALGS mutation; they are not simply due to JAG1 haploinsufficiency that is caused by a heterozygous knockout.

\section{Discussion}

We developed a system that differentiates human iPSCs into HOs in response to sequential changes in added growth factors. Four aspects of this system are particularly noteworthy. First, HOs contain 2 types of cells that are organized into a complex structure: it has sheets of hepatocytes, and the cells expressing cholangiocyte markers are organized into epithelia that surround the lumina of bile duct-like structures. Second, since virtually all (>97\%) of the cells express endodermal (SOX17) or hepatoblast (TBX3) markers at the appropriate developmental stage, this system is quite efficient at inducing organoid formation. Of note, TBX3 is expressed by a subpopulation of proliferating and self-renewing cells in the liver that are responsible for homeostatic hepatocyte renewal (46). Third, the iPSCs differentiate into HOs through stages that resemble liver during its embryonic development. Fourth, the organoids have a regenerative property that is characteristic of human liver. Their regenerative capacity is of importance. A recent review indicated that a major challenge for the human organoid field was scalability (47). The regenerative method described here addresses the scalability issue: at least 50 secondary HOs can be quickly produced from a single primary HO.

This system represents a substantial advance over the prior in vitro methods used to model human liver diseases or those used to produce liver organoids. iPSCs have previously been used to model some aspects of inherited metabolic disorders of the liver (48). Cholangiocytes have been produced from human embryonic stem cells (49) and from iPSCs (50), but different conditions are required for production of hepatocytes in the latter system. However, the development of a complex organ like the liver cannot be understood by analysis of a single type of cell, nor can the pathogenesis of genetic diseases affecting liver 
development be fully modeled using individual cells. Liver organoids have recently been produced from progenitor cells obtained from human liver tissue (31). However, human liver tissue is difficult to obtain and is available in very limited quantities. In contrast, very large numbers of iPSCs can easily be produced from readily accessible cell types. Moreover, we demonstrated that genome engineering could introduce human disease-causing mutations into iPSCs whose functional consequences can be analyzed in detail in HOs. Hence, the ability to produce HOs from iPSCs should dramatically expand the range of genetic diseases affecting liver that can be analyzed in vitro.

As a first example, we show how analysis of developing organoids can provide new insight into the pathogenesis of ALGS liver disease. ALGS patients are born with an intact biliary system (51), but the ratio of bile ducts to liver tissue quickly declines with age after birth, which leads to cholestasis and presentation with ALGS liver disease $(7,52,53)$. The proximal branches of the biliary tree are formed in embryonic liver from a single layer of bipotent precursor cells that surround the portal vein (the ductal plate), which are progressively remodeled to generate intrahepatic bile ducts (54), and this requires an interaction with surrounding mesodermal tissues (55). However, the liver enlarges 3 -fold during the postnatal period, which is when ALGS liver abnormalities first appear. Postnatal liver growth is mediated by an increase in the number of peripheral liver lobules, and this requires elongation and branching of the portal tracts (38). The inability to do this during the postnatal period generates the paucity of bile ducts in ALGS liver. Consistent with this, the extent of bile duct paucity varies with location in ALGS liver (7, 52,53 ), and is most severe in the liver periphery (56). Based upon analysis of ALGS liver tissue, it has been proposed that altered Notch signaling impairs the formation of the distal branches of the biliary tree during the postnatal period in ALGS liver (57). We propose that the ALGS mutation specifically affects postnatal liver development, because this occurs when the liver precursor cells are no longer in contact with surrounding mesodermal tissue. These features of ALGS liver disease are recapitulated in ALGS organoids: (a) ALGS iPSCs develop normally through the hepatoblast stage, and (b) mature hepatocytes are generated; (c) but the development of cholangiocytes and bile ductular structures is impaired, and (d) ALGS organoids can not regenerate secondary organoids. While the cystic structures lined by hepatocytes formed in ALGS organ cultures do not appear in ALGS liver, the cysts formed in the organoid cultures reflects the impaired ability of ALGS hepatoblasts to differentiate into cholangiocytes and to form bile ducts. We also found that $J A G 1$ mRNA expression, which peaks just before the bipotent hepatoblasts differentiate into cholangiocytes, is dynamically regulated during organoid formation. The JAG1 mutations produce liver pathology by disrupting the dynamic pattern of $J A G 1$ mRNA expression. Moreover, JAG1 is coexpressed with CK19 in developing organoids, and the bile duct-like tubules develop within the areas surrounded by JAG1-expressing cells. These findings are consistent with the inhibitory effect that NOTCH1 signaling has on hepatocyte proliferation (58) and in promoting intrahepatic bile duct development $(13,59)$. Given the composition of the cells within the HOs, immune-mediated destruction or inflammation cannot occur within developing organoid cultures. Our HO results indicate JAG1-expressing hepatoblasts appear to be the primary regulator of bile tubule formation in postnatal liver. This is consistent with recent evidence demonstrating that stem cells can provide feed-forward regulation via the Notch pathway on the downstream development of differentiated cells (60).

Our finding that $J A G 1$ haploinsufficiency alone does not produce pathology in liver organoids reveals important information about the pathogenesis of ALGS. At first, the dominant-negative effect exhibited by the $J A G 1$ mutation studied here may appear to differ from what has been observed in humans and mice. Since some ALGS subjects have a deletion of the entire JAG1 gene (24-26), others have concluded that ALGS pathology results from $J A G 1$ haploinsufficiency. The appearance of a cholangiopathy in $J A G 1^{+/-}$ heterozygous-knockout mice led to the same conclusion (23). However, haploinsufficient (Jag $1^{+/-}$) mice do not develop liver pathology $(21,22)$. Defects in bile duct development were only noted only after a mutation

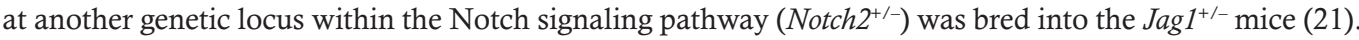
In another murine study, bile duct paucity only developed after Jag $1^{+/-}$heterozygosity was placed on a particular murine genetic background (23). The requirement for a particular strain background also indicates that another genetic factor (i.e., within the permissive strain background) was required for liver disease to develop in $\mathrm{Jag}^{+/-}$haploinsufficient mice. Hence, all of the murine data clearly indicate that Jag $1^{+/-}$haploinsufficiency alone does not produce ALGS liver disease; a second genetic effector is required for ALGS disease expression in the presence of JAG1 haploinsufficiency. Similarly, in humans, only a minority $(\sim 20 \%)$ of JAG1 mutation-positive relatives of ALGS subjects develop clinical features of ALGS (5). This variabil- 
ity in ALGS disease expression also indicates that some ALGS mutations require another genetic factor for ALGS pathology to appear in human subjects. Also of importance, only a small minority ( 7\%) of ALGS in human subjects results from a gene deletion (4). Hence, in the minority of human ALGS disease, which is associated with $J A G 1$ haploinsufficiency, it is likely that a second genetic factor is required for disease expression. For the vast majority of those with other types of $J A G 1$ mutations, our organoid results indicate that ALGS liver disease is likely to be mediated by a dominant-negative effector mechanism.

Liver organoids were also used to investigate the basis for the variability in the clinical features caused by $J A G 1$ mutations. While genetic modifiers at other sites in the genome do contribute to this variability (5), our results demonstrate that the type of $J A G 1$ mutation itself has a significant affect on the appearance of liver disease. When different $J A G 1$ mutations were engineered onto the same genetic background, the mutations (Cys829X and ALGS2) found in subjects with severe liver disease impaired organoid development, while a mutation (Gly274Asp) causing TOF in subjects without liver disease did not impair organoid formation. The combined use of iPSCs and genome editing technologies has enabled revolutionary advances in many areas (61). Our results indicate how this organoid system and genome editing can be jointly used to determine how human disease-causing mutations affect organ development and the pathogenesis of human genetic diseases.

\section{Methods}

Further details about the experimental procedures, including antibody and RT-PCR primer information, can be found in the supplemental material.

iPSC cultures. Human iPSC lines C1, C2, and C3 were maintained as monolayer clones under feeder-free conditions on human-ES-cell-qualified Matrigel (BD Biosciences) in TeSR-E8 (StemCell Technologies). Cells were routinely passaged at a ratio of 1:10 every 4 to 6 days using 5 mM EDTA (Invitrogen).

$3 D$ human $\mathrm{HO}$ culture. For production of HO1s, iPSCs were dissociated to single cells in buffer with $5 \mathrm{mM}$ EDTA and $10 \mathrm{mM}$ Y-27632 (Santa Cruz Biotechnology), and were reaggregated in low-cell-adhesion 60-mm dishes (Corning) in endoderm differentiation medium (10 cells/dish) containing $10 \mathrm{mM}$ Y-27632. The endoderm differentiation medium consisted of DMEM/F12 plus ITS (GIBCO) supplemented with $0.1 \mathrm{mM}$ nonessential amino acids and $1 \mathrm{mM}$ pyruvate. On days 0 to 3, activin-A (100 ng/ $\mathrm{ml})$ and BMP4 $(10 \mathrm{ng} / \mathrm{ml})$ were added to the chemically defined endoderm differentiation medium. For endoderm patterning and for the generation of foregut spheroids, Matrigel (BD Biosciences, growth factor reduced [GFR]) was added to RPMI and B27 medium to a final v/v concentration of $2 \%$ from days 3 to 9 . From days 3 to $6,50 \mathrm{ng} / \mathrm{ml} \mathrm{FGF10} \mathrm{(Peprotech)} \mathrm{was} \mathrm{added} \mathrm{to} \mathrm{the} \mathrm{medium.} \mathrm{From} \mathrm{days} 6$ to $9,10 \mathrm{ng} /$ $\mathrm{ml} \mathrm{FGF} 10$ and $10 \mathrm{ng} / \mathrm{ml} \mathrm{BMP} 4$ were added to the medium. After day 9, Matrigel (final 1\% v/v concentration) was added to the organoid growth and differentiation medium (HCM, Lonza), which was supplemented with HGF (50 ng/ml), $50 \mathrm{ng} / \mathrm{ml}$ oncostatin $\mathrm{M}$, and $10 \mu \mathrm{M}$ dexamethasone (Sigma-Aldrich). On day 20, the organoids were collected and reseeded for further differentiation and characterization.

HO2 culture. The cells in control and ALGS HO1 cultures (day 20 to 50) were dissociated by digestion with $1 \mathrm{mg} / \mathrm{ml}$ collagenase for 30 minutes, and then with $0.25 \%$ trypsin-EDTA for 10 minutes. The cells were collected by centrifugation at $200 \mathrm{~g}$ for 3 minutes, resuspended in $50 \mu 1$ of GFR-Matrigel, and plated at 1,000 cells per well in a 24-well plate. After Matrigel solidification, $1 \mathrm{ml}$ of growth media was added, and the cells were cultured for 6 days. The growth media consisted of RPMI plus B27 (GIBCO) medium with the following growth factors: $250 \mathrm{nM}$ LDN-193189 (iBMP), $3 \mu \mathrm{M}$ CHIR99021, $10 \mu \mathrm{M}$ A83-01 (iTGF), $100 \mathrm{ng} / \mathrm{ml} \mathrm{EGF}, 10 \mathrm{ng} / \mathrm{ml} \mathrm{FGF10,} \mathrm{and} 20 \mathrm{ng} / \mathrm{ml}$ HGF. The cells were then cultured for 6 more days in a differentiation medium, which consisted of HCM medium supplemented with $10 \mu \mathrm{M}$ DAPT (iNOTCH), $10 \mathrm{ng} / \mathrm{ml}$ oncostatin $\mathrm{M}, 20 \mathrm{ng} / \mathrm{ml} \mathrm{HGF}, 10 \mu \mathrm{M}$ dexamethasone, and $10 \mathrm{ng} / \mathrm{ml} \mathrm{BMP} 4$.

Statistics. Data were pooled from at least 3 independent sets of experiments, unless otherwise indicated, and are presented as the mean \pm SD. All statistical analyses were carried out using SPSS 11 (SPSS). Comparisons of mean values were analyzed by Student's $t$ test (2 sided) or 1-way ANOVA followed by the post-hoc Dunnett's test for experiments with more than 2 groups (Levene's tests for equal variance). Dunnett's T3 test was used as post-hoc test comparison for the analysis of unequal variances (Welch's and Brown-Forsythe's test). Differences were considered statistically significant at $P$ less than 0.05 . Graphing was performed using SigmaPlot and R software.

Study approval. The samples were obtained according to a protocol (number 10368) that was approved by the Institutional Review Board at Stanford. Additional experimental details are provided in the Supplemental Materials and Methods. 


\section{Author contributions}

YG, JS, and GP wrote the manuscript. YG, DX, PMG, UE, MH, SM, MW, and TN performed experiments. MZ analyzed the RNA-Seq data. GE obtained patient samples.

\section{Acknowledgments}

We thank Elizabeth B. Rand from Children's Hospital of Philadelphia for providing the ALGS liver tissue samples. This study was supported by a transformative award (5R01DK09099205) and other funding (1R01DK10218201A1) awarded to G.P. from the NIH/NIDDK. This study was also supported by funding awarded to J.S. from the following organizations: the Lucile Packard Foundation for Children's Health, the Stanford NIH-NCATS-CTSA UL1 TR001085, the Child Health Research Institute of Stanford University, and 1R01CA114102 from the NIH/NCI. The authors would like to thank Louis Saddic, Anne-Flore Zmoos, and Gavin Wang for their help in the initial stages of this study; and Carlos Esquivel and Waldo Concepcion for providing surgical samples.

Address correspondence to: Gary Peltz, 300 Pasteur Drive, Room L232 Stanford, California 94305, USA.Email: gpeltz@stanford.edu.

1. Zaret KS. Genetic programming of liver and pancreas progenitors: lessons for stem-cell differentiation. Nat Rev Genet. 2008;9(5):329-340.

2. Oda T, et al. Mutations in the human Jagged1 gene are responsible for Alagille syndrome. Nat Genet. 1997;16(3):235-242.

3. Li L, et al. Alagille syndrome is caused by mutations in human Jagged1, which encodes a ligand for Notch1. Nat Genet. $1997 ; 16(3): 243-251$

4. Penton AL, Leonard LD, Spinner NB. Notch signaling in human development and disease. Semin Cell Dev Biol. 2012;23(4):450-457.

5. Kamath BM, Bason L, Piccoli DA, Krantz ID, Spinner NB. Consequences of JAG1 mutations. J Med Genet. 2003;40(12):891-895.

6. Fallot ELA. Contribution a` 1'anatomie pathologique de la maladie bleue (cyanose cardiaque). Marsaille Medical. 1881;25:77-95.

7. Emerick KM, Rand EB, Goldmuntz E, Krantz ID, Spinner NB, Piccoli DA. Features of Alagille syndrome in 92 patients: frequency and relation to prognosis. Hepatology. 1999;29(3):822-829.

8. Anderson RH, Spicer DE, Giroud JM, Mohun TJ. Tetralogy of Fallot: nosological, morphological, and morphogenetic considerations. Cardiol Young. 2013;23(6):858-866.

9. Krantz ID, et al. Jagged 1 mutations in patients ascertained with isolated congenital heart defects. Am J Med Genet. 1999;84(1):56-60

10. Artavanis-Tsakonas S, Matsuno K, Fortini ME. Notch signaling. Science. 1995;268(5208):225-232.

11. Guruharsha KG, Kankel MW, Artavanis-Tsakonas S. The Notch signalling system: recent insights into the complexity of a conserved pathway. Nat Rev Genet. 2012;13(9):654-666.

12. Fleming RJ, Purcell K, Artavanis-Tsakonas S. The NOTCH receptor and its ligands. Trends Cell Biol. 1997;7(11):437-441.

13. Kodama Y, Hijikata M, Kageyama R, Shimotohno K, Chiba T. The role of notch signaling in the development of intrahepatic bile ducts. Gastroenterology. 2004;127(6):1775-1786.

14. Lorent $\mathrm{K}$, et al. Inhibition of Jagged-mediated Notch signaling disrupts zebrafish biliary development and generates multi-organ defects compatible with an Alagille syndrome phenocopy. Development. 2004;131(22):5753-5766.

15. Zender S, et al. A critical role for notch signaling in the formation of cholangiocellular carcinomas. Cancer Cell. 2013;23(6):784-795.

16. Sekiya S, Suzuki A. Intrahepatic cholangiocarcinoma can arise from Notch-mediated conversion of hepatocytes. J Clin Invest. 2012;122(11):3914-3918.

17. Crawford JM. Development of the intrahepatic biliary tree. Semin Liver Dis. 2002;22(3):213-226.

18. Crosnier C, et al. JAGGED1 gene expression during human embryogenesis elucidates the wide phenotypic spectrum of Alagille syndrome. Hepatology. 2000;32(3):574-581.

19. Hofmann JJ, Zovein AC, Koh H, Radtke F, Weinmaster G, Iruela-Arispe ML. Jagged1 in the portal vein mesenchyme regulates intrahepatic bile duct development: insights into Alagille syndrome. Development. 2010;137(23):4061-4072.

20. Sparks EE, Huppert KA, Brown MA, Washington MK, Huppert SS. Notch signaling regulates formation of the three-dimensional architecture of intrahepatic bile ducts in mice. Hepatology. 2010;51(4):1391-1400.

21. McCright B, Lozier J, Gridley T. A mouse model of Alagille syndrome: Notch2 as a genetic modifier of Jag1 haploinsufficiency Development. 2002;129(4):1075-1082.

22. Xue Y, et al. Embryonic lethality and vascular defects in mice lacking the Notch ligand Jagged1. Hum Mol Genet. 1999;8(5):723-730.

23. Thakurdas SM, et al. Jagged1 heterozygosity in mice results in a congenital cholangiopathy which is reversed by concomitant deletion of one copy of Poglut1 (Rumi). Hepatology. 2016;63(2):550-565.

24. Deleuze JF, Hazan J, Dhorne S, Weissenbach J, Hadchouel M. Mapping of microsatellite markers in the Alagille region and screening of microdeletions by genotyping 23 patients. Eur J Hum Genet. 1994;2(3):185-190.

25. Desmaze C, Deleuze JF, Dutrillaux AM, Thomas G, Hadchouel M, Aurias A. Screening of microdeletions of chromosome 20 in patients with Alagille syndrome. J Med Genet. 1992;29(4):233-235.

26. Deleuze JF, et al. Deleted chromosome 20 from a patient with Alagille syndrome isolated in a cell hybrid through leucine transport selection: study of three candidate genes. Mamm Genome. 1994;5(11):663-669.

27. Spinner NB, Colliton RP, Crosnier C, Krantz ID, Hadchouel M, Meunier-Rotival M. Jagged1 mutations in alagille syndrome. Hum Mutat. 2001;17(1):18-33. 
28. Grochowski CM, Loomes KM, Spinner NB. Jagged1 (JAG1): Structure, expression, and disease associations. Gene. 2016;576(1 Pt 3):381-384.

29. Lu F, Morrissette JJ, Spinner NB. Conditional JAG1 mutation shows the developing heart is more sensitive than developing liver to JAG1 dosage. Am J Hum Genet. 2003;72(4):1065-1070.

30. Hannan NR, Segeritz CP, Touboul T, Vallier L. Production of hepatocyte-like cells from human pluripotent stem cells. Nat Protoc. $2013 ; 8(2): 430-437$.

31. Huch M, et al. Long-term culture of genome-stable bipotent stem cells from adult human liver. Cell. 2015;160(1-2):299-312.

32. Nakano T, et al. Self-formation of optic cups and storable stratified neural retina from human ESCs. Cell Stem Cell. 2012;10(6):771-785.

33. Dong PD, et al. Fgf10 regulates hepatopancreatic ductal system patterning and differentiation. Nat Genet. 2007;39(3):397-402

34. Suzuki A, Sekiya S, Büscher D, Izpisúa Belmonte JC, Taniguchi H. Tbx3 controls the fate of hepatic progenitor cells in liver development by suppressing p19ARF expression. Development. 2008;135(9):1589-1595.

35. Shiojiri N. The origin of intrahepatic bile duct cells in the mouse. J Embryol Exp Morphol. 1984;79:25-39.

36. Nishimura T, et al. Using chimeric mice with humanized livers to predict human drug metabolism and a drug-drug interaction. J Pharmacol Exp Ther. 2013;344(2):388-396.

37. Landing BH, Wells TR. Considerations of Some Architectural Properties of the Biliary Tree and Liver in Childhood. In: Abramowsky CR, Rosenberg BJ eds. Transplantation Pathology-Hepatic Morphogenesis. Basel: Kruger; 1981:122-42.

38. Landing BH, Wells TR. Considerations of some architectural properties of the biliary tree and liver in childhood. Perspect Pediatr Pathol. 1991;14:122-142.

39. Furuyama K, et al. Continuous cell supply from a Sox9-expressing progenitor zone in adult liver, exocrine pancreas and intestine. Nat Genet. 2011;43(1):34-41.

40. Boulter L, et al. Macrophage-derived Wnt opposes Notch signaling to specify hepatic progenitor cell fate in chronic liver disease. Nat Med. 2012;18(4):572-579.

41. D’Souza B, Meloty-Kapella L, Weinmaster G. Canonical and non-canonical Notch ligands. Curr Top Dev Biol. 2010;92:73-129.

42. Hammad S, et al. Protocols for staining of bile canalicular and sinusoidal networks of human, mouse and pig livers, three-dimensional reconstruction and quantification of tissue microarchitecture by image processing and analysis. Arch Toxicol. 2014;88(5):1161-1183.

43. Iso T, Kedes L, Hamamori Y. HES and HERP families: multiple effectors of the Notch signaling pathway. J Cell Physiol. 2003;194(3):237-255.

44. Gigliozzi A, et al. Molecular identification and functional characterization of Mdr1a in rat cholangiocytes. Gastroenterology. 2000;119(4):1113-1122.

45. Eldadah ZA, et al. Familial Tetralogy of Fallot caused by mutation in the jagged1 gene. Hum Mol Genet. 2001;10(2):163-169.

46. Sia D, et al. Massive parallel sequencing uncovers actionable FGFR2-PPHLN1 fusion and ARAF mutations in intrahepatic cholangiocarcinoma. Nat Commun. 2015;6:6087.

47. McCauley HA, Wells JM. Pluripotent stem cell-derived organoids: using principles of developmental biology to grow human tissues in a dish. Development. 2017;144(6):958-962.

48. Rashid ST, et al. Modeling inherited metabolic disorders of the liver using human induced pluripotent stem cells. J Clin Invest. 2010;120(9):3127-3136.

49. Ogawa M, et al. Directed differentiation of cholangiocytes from human pluripotent stem cells. Nat Biotechnol. 2015;33(8):853-861.

50. Sampaziotis F, et al. Cholangiocytes derived from human induced pluripotent stem cells for disease modeling and drug validation. Nat Biotechnol. 2015;33(8):845-852.

51. Strazzabosco M, Fabris L. Development of the bile ducts: essentials for the clinical hepatologist. J Hepatol. 2012;56(5):1159-1170

52. Dahms BB, et al. Arteriohepatic dysplasia in infancy and childhood: a longitudinal study of six patients. Hepatology. 1982;2(3):350-358.

53. Kahn EI, et al. Arteriohepatic dysplasia. II. Hepatobiliary morphology. Hepatology. 1983;3(1):77-84.

54. Carpentier R, et al. Embryonic ductal plate cells give rise to cholangiocytes, periportal hepatocytes, and adult liver progenitor cells. Gastroenterology. 2011;141(4):1432-8, 1438.e1

55. Crawford LW, Foley JF, Elmore SA. Histology atlas of the developing mouse hepatobiliary system with emphasis on embryonic days 9.5-18.5. Toxicol Pathol. 2010;38(6):872-906.

56. Libbrecht L, Spinner NB, Moore EC, Cassiman D, Van Damme-Lombaerts R, Roskams T. Peripheral bile duct paucity and cholestasis in the liver of a patient with Alagille syndrome: further evidence supporting a lack of postnatal bile duct branching and elongation. Am J Surg Pathol. 2005;29(6):820-826.

57. Fabris L, et al. Analysis of liver repair mechanisms in Alagille syndrome and biliary atresia reveals a role for notch signaling Am J Pathol. 2007;171(2):641-653.

58. Croquelois A, et al. Inducible inactivation of Notch1 causes nodular regenerative hyperplasia in mice. Hepatology. 2005;41(3):487-496.

59. Jörs S, et al. Lineage fate of ductular reactions in liver injury and carcinogenesis. J Clin Invest. 2015;125(6):2445-2457.

60. Pardo-Saganta A, et al. Parent stem cells can serve as niches for their daughter cells. Nature. 2015;523(7562):597-601.

61. Hockemeyer D, Jaenisch R. Induced pluripotent stem cells meet genome editing. Cell Stem Cell. 2016;18(5):573-586. 